\title{
The fleet size and mix location-routing problem with time windows: Formulations and a heuristic algorithm
}

\author{
Çağrı Koç§ $\quad$ Tolga Bektaş $\quad$ Ola Jabali ${ }^{\S} \quad$ Gilbert Laporte \\ §outhampton Business School \\ and \\ Centre for Operational Research, Management Science and Information Systems (CORMSIS) \\ University of Southampton, Southampton, United Kingdom \\ e-mail: $\{$ C.Koc, T.Bektas\}@soton.ac.uk \\ ${ }^{\dagger}$ CIRRELT and HEC Montréal, \\ Montréal, Canada \\ e-mail: Ola.Jabali@hec.ca \\ ${ }^{\ddagger}$ CIRRELT, Canada Research Chair in Distribution Management and HEC Montréal \\ Montréal, Canada \\ e-mail: Gilbert.Laporte@cirrelt.ca
}

\begin{abstract}
This paper introduces the fleet size and mix location-routing problem with time windows (FSMLRPTW) which extends the location-routing problem by considering a heterogeneous fleet and time windows. The main objective is to minimize the sum of vehicle fixed cost, depot cost and routing cost. We present mixed integer programming formulations, a family of valid inequalities and we develop a powerful hybrid evolutionary search algorithm (HESA) to solve the problem. The HESA successfully combines several metaheuristics and offers a number of new advanced efficient procedures tailored to handle heterogeneous fleet dimensioning and location decisions. We evaluate the strengths of the proposed formulations with respect to their ability to find optimal solutions. We also investigate the performance of the HESA. Extensive computational experiments on new benchmark instances have shown that the HESA is highly effective on the FSMLRPTW.

Keywords. location-routing; heterogeneous fleet; time windows; mixed integer programming; genetic algorithm.
\end{abstract}

\section{Introduction}

The design of distribution networks is critical for most companies because it usually requires a major capital outlay. Two major types of decisions intervene in this process, namely determining the locations of depots, and designing vehicle routes supplying customers from these depots. In the classical Facility Location Problem (FLP) (Balinski, 1965), it is assumed that each customer is served individually through a direct shipment, which makes sense when customer demands are close to the vehicle capacity. However, there exist several situations where customer demands can be consolidated. In such contexts, the FLP and Vehicle Routing Problem (VRP) should be solved jointly. The idea of combining location and routing decisions was put forward more than fifty years ago (Von Boventer, 1961) and has given rise to a rich research known as the Location-Routing Problem (LRP) (see Laporte, 1988; Min et al., 1998; Nagy and Salhi, 2007; Prodhon and Prins, 2014; Albareda-Sambola, 2015; Drexl and Schneider, 2015, for reviews). Applications of the LRP arise 
in areas as diverse as food and drink distribution, parcel delivery and telecommunication network design. Many algorithms, mostly heuristics, have been developed for the LRP and its variations over the past fifty years, including some population metaheuristics (Prins et al., 2006b; Duhamel et al., 2010), neighborhoodbased metaheuristics (Prins et al., 2006a; Duhamel et al., 2011), simulated annealing (Karaoglan et al., 2012) and adaptive large neighborhood search (ALNS) algorithms (Hemmelmayr et al., 2012).

According to Hoff et al. (2010), heterogeneous fleets are more common in real-world distribution problems than homogeneous ones. The Fleet Size and Mix VRP with Time Windows (FSMVRPTW), introduced by Liu and Shen (1999), is an LRP with a single depot, a heterogeneous fleet and time windows. Many heuristics have also been developed for the FSMVRPTW (see Irnich et al., 2014), including a two-stage construction heuristic (Liu and Shen, 1999), a sequential construction heuristic (Dullaert et al., 2002), a multi-start parallel regret construction heuristic (Dell'Amico et al., 2007), a three-phase multi-restart deterministic annealing metaheuristic (Bräysy et al., 2008), a hybrid metaheuristic combining a threshold acceptance heuristic with a guided local search (Bräysy et al., 2009), an adaptive memory programming algorithm (Repoussis and Tarantilis, 2010), and a genetic algorithm using a unified component based solution framework (Vidal et al., 2014). This problem and a number of its variations are reviewed by Baldacci et al. (2008) and Baldacci and Mingozzi (2009).

To our knowledge, a number of studies indirectly consider heterogeneous fleets in an LRP context but without taking time windows into account (Ambrosino et al., 2009; Wu et al., 2010). Berger et al. (2007) only consider a distance constraint. Therefore, combining heterogeneous fleets and time windows in the LRP is done here for the first time. We believe there is methodological interest in solving the Fleet Size and Mix Location-Routing Problem with Time Windows (FSMLRPTW).

In the FSMLRPTW, one considers a fleet of vehicles with various capacities and vehicle-related costs, as well as a set of potential depots with opening costs and capacities, and a set of customers with known demands and time windows. The FSMLRPTW consists of opening a subset of depots, assigning customers to them and determining a set of routes for heterogeneous vehicles such that all vehicles start and end their routes at their depot, each customer is visited exactly once by a vehicle within a prespecified time window, and the load of each vehicle does not exceed its capacity. The objective is to minimize the total cost which is made up of three components: the depot operating cost, the fixed cost of the vehicles, and the total travel costs of the vehicles. It is assumed that these costs are scaled over the same time horizon.

The contributions of this paper are as follows. We introduce the FSMLPRTW as a new LRP variant. We develop a hybrid evolutionary search algorithm (HESA) with the introduction of several algorithmic pro- 
cedures specific to the FSMLRPTW. Namely, we introduce the location-heterogeneous adaptive large neighborhood search (L-HALNS) procedure equipped with a range of several new operators as the main EDUCATION procedure within the search. We also propose an INITIALIZATION procedure to create initial solutions, and a PARTITION procedure for offspring solutions. Finally, we develop a new diversification scheme through the MUTATION procedure of solutions.

The remainder of this paper is structured as follows. Section 2 formally defines the problem and provides integer programming formulations together with valid inequalities. Section 3 presents a detailed description of the HESA. Computational experiments are provided in Section 4, and conclusions follow in Section 5.

\section{Formulations for the fleet size and mix location-routing problem with time windows}

In this section, we first define the FSMLRPTW, and then present several integer programming formulations and valid inequalities to strengthen them.

\subsection{Notation and problem definition}

The FSMLRPTW is defined on a complete directed graph $\mathcal{G}=(\mathcal{N}, \mathcal{A})$, where $\mathcal{N}=\mathcal{N}_{0} \cup \mathcal{N}_{c}$ is a set of nodes in which $\mathcal{N}_{0}$ and $\mathcal{N}_{c}$ represent the potential depot and customer nodes, respectively, and $\mathcal{A}=\{(i, j): i \in$ $\mathcal{N}, j \in \mathcal{N}\} \backslash\left\{(i, j): i \in \mathcal{N}_{0}, j \in \mathcal{N}_{0}, i \neq j\right\}$ is the set of arcs. Each $\operatorname{arc}(i, j) \in \mathcal{A}$ has a nonnegative distance $c_{i j}$. Here, the terms distance, travel time and travel cost are used in interchangeably. Each customer $i \in \mathcal{N}_{c}$ has a positive demand $q_{i}$. A storage capacity $D^{k}$ and a fixed opening $\operatorname{cost} g^{k}$ are associated with each potential $\operatorname{depot} k \in \mathcal{N}_{0}$. The index set of vehicle types is denoted by $\mathcal{H}$. Let $Q^{h}$ and $f^{h}$ denote the capacity and fixed dispatch cost of a vehicle of type $h \in \mathcal{H}$. Furthermore, $s_{i}$ corresponds to the service time of node $i \in \mathcal{N}_{c}$, which must start within the time window $\left[a_{i}, b_{i}\right]$. If a vehicle arrives at customer $i \in \mathcal{N}_{c}$ before time $a_{i}$, it waits until $a_{i}$ to start servicing the customer.

To formulate the FSMLRPTW, we define the following decision variables. Let $x_{i j}^{h}$ be equal to 1 if vehicle of type $h$ travels directly from node $i$ to node $j$ and to 0 otherwise. Let $y_{k}$ be equal to 1 if depot $k$ is opened and to 0 otherwise. Let $z_{i k}$ be equal to 1 if customer $i$ is assigned to depot $k$ and to 0 otherwise. Let $u_{i j}^{h}$ be the total load of vehicle of type $h$ immediately after visiting node $i$ and traveling directly to node $j$. Let $f_{i j}$ 
be the total load of the vehicle while traversing arc $(i, j) \in \mathcal{A}$, which is the aggregation of the $u_{i j}^{h}$ variables over $\mathcal{H}$. Let $t_{i}^{h}$ be the time at which a vehicle of type $h$ starts serving at node $i$. Let $t_{i}$ be the time at which service starts at node $i \in \mathcal{N}$, which is the aggregation of the $t_{i}^{h}$ variables.

\subsection{Integer programming formulations}

The integer linear programming formulation of the problem, denoted by $E_{1}$, is then:

$$
\left(E_{1}\right) \text { Minimize } \sum_{k \in \mathcal{N}_{0}} g^{k} y_{k}+\sum_{h \in \mathcal{H}} \sum_{k \in \mathcal{N}_{0}} \sum_{i \in \mathcal{N}_{c}} f^{h} x_{k i}^{h}+\sum_{h \in \mathcal{H}} \sum_{(i, j) \in \mathcal{A}} c_{i j} x_{i j}^{h}
$$

subject to

$$
\begin{aligned}
& \sum_{h \in \mathcal{H}} \sum_{j \in \mathcal{N}} x_{i j}^{h}=1 \quad i \in \mathcal{N}_{c} \\
& \sum_{h \in \mathcal{H}} \sum_{j \in \mathcal{N}} x_{j i}^{h}=\sum_{h \in \mathcal{H}} \sum_{j \in \mathcal{N}} x_{i j}^{h} \quad i \in \mathcal{N} \\
& \sum_{h \in \mathcal{H}} \sum_{j \in \mathcal{N}} u_{j i}^{h}-\sum_{h \in \mathcal{H}} \sum_{j \in \mathcal{N}} u_{i j}^{h}=q_{i} \quad i \in \mathcal{N}_{c} \\
& u_{i j}^{h} \leq Q^{h} x_{i j}^{h} \quad i \in \mathcal{N}_{0}, j \in \mathcal{N}, i \neq j, h \in \mathcal{H} \\
& \sum_{h \in \mathcal{H}} \sum_{j \in \mathcal{N}_{c}} u_{k j}^{h}=\sum_{j \in \mathcal{N}_{c}} z_{j k} q_{j} \quad k \in \mathcal{N}_{0} \\
& \sum_{h \in \mathcal{H}} \sum_{j \in \mathcal{N}_{c}} u_{j k}^{h}=0 \quad k \in \mathcal{N}_{0} \\
& u_{i j}^{h} \leq\left(Q^{h}-q_{i}\right) x_{i j}^{h} \quad i \in \mathcal{N}_{c}, j \in \mathcal{N}, h \in \mathcal{H} \\
& u_{i j}^{h} \geq q_{j} x_{i j}^{h} \quad i \in \mathcal{N}, j \in \mathcal{N}_{c}, h \in \mathcal{H} \\
& \sum_{i \in \mathcal{N}_{c}} q_{i} z_{i k} \leq D^{k} y_{k} \quad k \in \mathcal{N}_{0} \\
& \sum_{k \in \mathcal{N}_{0}} z_{i k}=1 \quad i \in \mathcal{N}_{c} \\
& x_{i j}^{h}+\sum_{q \in \mathcal{H}, q \neq h} \sum_{l \in \mathcal{N}, j \neq l} x_{j l}^{q} \leq 1 \quad i \in \mathcal{N}, j \in \mathcal{N}_{c}, i \neq j, h \in \mathcal{H} \\
& \sum_{h \in \mathcal{H}} x_{i k}^{h} \leq z_{i k} \quad k \in \mathcal{N}_{0}, i \in \mathcal{N}_{c} \\
& \sum_{h \in \mathcal{H}} x_{k i}^{h} \leq z_{i k} \quad k \in \mathcal{N}_{0}, i \in \mathcal{N}_{c} \\
& \sum_{h \in \mathcal{H}} x_{i j}^{h}+z_{i k}+\sum_{m \in \mathcal{N}_{0}, m \neq k} z_{j m} \leq 2 \quad k \in \mathcal{N}_{0},(i, j) \in \mathcal{N}_{c}, i \neq j
\end{aligned}
$$




$$
\begin{array}{rl}
t_{i}^{h}-t_{j}^{h}+s_{i}+c_{i j} \leq M\left(1-x_{i j}^{h}\right) & i \in \mathcal{N}, j \in \mathcal{N}_{c}, i \neq j, h \in \mathcal{H} \\
a_{i} \leq t_{i}^{h} \leq b_{i} & i \in \mathcal{N}, h \in \mathcal{H} \\
x_{i j}^{h} \in\{0,1\} & (i, j) \in \mathcal{N}, h \in \mathcal{H} \\
z_{i k} \in\{0,1\} & k \in \mathcal{N}_{0}, i \in \mathcal{N}_{c} \\
y_{k} \in\{0,1\} & k \in \mathcal{N}_{0} \\
u_{i j}^{h} \geq 0 & (i, j) \in \mathcal{N}, h \in \mathcal{H} \\
t_{i}^{h} \geq 0 & i \in \mathcal{N}_{c}, h \in \mathcal{H} .
\end{array}
$$

The objective function (1) minimizes the total cost including depot fixed cost, vehicle fixed cost and variable travel cost. Constraints (2) and (3) are degree constraints; in particular constraints (2) guarantee that each customer must be visited exactly once, and constraints (3) ensure that entering and leaving arcs to each node are equal. Constraints (4) imply that the demand of each customer is satisfied. Constraints (5) mean that the total load on any arc cannot exceed the capacity of the vehicle traversing it. Constraints (6) ensure that the total load of each depot is equal to the total demand of customers assigned to it. Note that constraints (6) strengthen the formulation, yet are not necessary. Constraints (7) state that the load on a vehicle returning to each depot must be equal to zero. Contraints (8) and (9) are bounding constraints for load variables. Constraints (10) guarantee that total demand supplied by a depot cannot exceed its capacity. Constraints (11) and (12) ensure that each customer is assigned to only one depot and to only one vehicle type, respectively. Constraints (13)-(15) forbid illegal routes, i.e., routes that do not start and end at the same depot. Constraints (16) and (17), where $M$ is a large number, enforce the time window restrictions. Constraints (18)-(22) define the domains of the decision variables.

The validity of constraints (13)-(15) was proven by Karaoglan et al. (2011). They prevent illegal routes starting at one depot and ending at another, the prevents the creation of infeasible routes $P=\left(v_{k_{1}}, v_{1}, v_{2}\right.$, $\left.\ldots, v_{s-1}, v_{s}, v_{l_{2}}\right)$ with $s$ customers $\left(k, l \in \mathcal{N}_{0}, k \neq l\right)$. Constraints (13) imply that $z_{v_{1}, k_{1}}=1$. Since $x_{v_{1}, v_{2}}=1$ and $z_{v_{1}, k_{1}}=1$, constraints (15) ensure that $z_{v_{2}, k_{1}}=1$ and $z_{v_{2}, k_{2}}$ cannot be equal to one.

Formulation $E_{1}$ shown by (1)-(22) is valid for the FSMLRPTW. Other valid formulations can be derived from $E_{1}$. Before defining these formulations, we provide several variations of $E_{1}$ by aggregating some of the variables or disaggregating some of the constraints. The reason is to either reduce the size of the formulation through aggregation of variables or to tighten the linear relaxation bound by disaggregating 
some of the constraints. First, $f_{i j}$ which is the aggregation of the $u_{i j}^{h}$ variables, is obtained as follows:

$$
\begin{array}{rl}
\sum_{j \in \mathcal{N}} f_{j i}-\sum_{j \in \mathcal{N}} f_{i j}=q_{i} & i \in \mathcal{N}_{c} \\
f_{i j} \leq \sum_{h \in \mathcal{H}} Q^{h} x_{i j}^{h} & i \in \mathcal{N}_{0}, j \in \mathcal{N}, i \neq j \\
\sum_{j \in \mathcal{N}_{c}} f_{k j}=\sum_{j \in \mathcal{N}_{c}} z_{j k} q_{j} & k \in \mathcal{N}_{0} \\
\sum_{j \in \mathcal{N}_{c}} f_{j k}=0 & k \in \mathcal{N}_{0} \\
f_{i j} \leq \sum_{h \in \mathcal{H}}\left(Q^{h}-q_{i}\right) x_{i j}^{h} & i \in \mathcal{N}_{c}, j \in \mathcal{N} \\
f_{i j} \geq q_{j} \sum_{h \in \mathcal{H}} x_{i j}^{h} & i \in \mathcal{N}_{, j} \in \mathcal{N}_{c} .
\end{array}
$$

Let $(f, x)$ be a solution satisfying constraints (23)-(28) and let $(u, x)$ be a solution satisfying constraints (2) and (4)-(9), where $f, u$ and $x$ are the respective vectors for variables $f_{i j}, u_{i j}^{h}$ and $x_{i j}^{h}$. Then, for each feasible $(u, x)$, there exists a feasible $(f, x)$ and vice versa. Due to (2), there exists an $h^{*} \in \mathcal{H}$ for which $x_{i j}^{h^{*}}=1$ and due to (8), there exists a $u_{i j}^{h^{*}} \geq 0$, implying $x_{i j}^{h}=u_{i j}^{h}=0$ for all other $h \in \mathcal{H} \backslash\left\{h^{*}\right\}$. Using these arguments, we have linked $f_{i j}$ to the original variables in the following way:

$$
f_{i j}=\sum_{h \in \mathcal{H}} u_{i j}^{h} \quad i, j \in \mathcal{N} .
$$

We can now derive constraints (23)-(28) from (4)-(9) by using definition (29). Constraints (23)-(28) are satisfied, since they are the same as (4)-(9), respectively.

Second, the aggregation of the $t_{i}^{h}$ variable is

$$
\begin{gathered}
t_{i}-t_{j}+s_{i}+c_{i j} \leq M\left(1-\sum_{h \in \mathcal{H}} x_{i j}^{h}\right) \quad i \in \mathcal{N}, j \in \mathcal{N}_{c}, i \neq j \\
a_{i} \leq t_{i} \leq b_{i} \quad i \in \mathcal{N} .
\end{gathered}
$$

Constraints (30)-(31) and (16)-(17) are equivalent. Due to (16) there exists $h^{*} \in \mathcal{H}$ for which $x_{i j}^{h^{*}}=1$, i.e., $x_{i j}^{h}=0$ for all $h \neq h^{*}, h \in \mathcal{H}$. An equivalent definition is

$$
t_{j}=\sum_{h \in \mathcal{H}} t_{j}^{h} \quad j \in \mathcal{N}
$$

We can now derive constraints (30)-(31) from (16)-(17) by using definition (32). Constraints (30)-(31) are satisfied, since they are the same as (16)-(17), respectively. 
Third, the disaggregation of the balance constraints (3) is

$$
\sum_{j \in \mathcal{N}} x_{j i}^{h}=\sum_{j \in \mathcal{N}} x_{i j}^{h} \quad h \in \mathcal{H}, i \in \mathcal{N}
$$

Constraints (33) are equivalent to constraints (3) and (12). Given that $i, j, k$ are on the same route, assume $h^{1}, h^{2} \in \mathcal{H}, x_{i j}^{h^{1}}=1$ and $x_{j k}^{h^{2}}=1$, which is valid for (3). But constraints (12) along with (3) only allow the use of the same vehicle on a route, i.e, $x_{i j}^{h^{1}}=1$ and $x_{j k}^{h^{1}}=1$. We can now derive constraints (33) from (3). Using (33) also makes (12) redundant. Constraints (33) ensure that $x_{i j}^{h^{1}}=1$ and $x_{j k}^{h^{1}}=1$ without using (12). Constraints (33) are satisfied, since they are the same as (3), respectively.

We now define the following four variations of $E_{1}$ as follows:

1) Formulation $E_{2}$ minimizes (1), subject to (2), (3), (23)-(28) and (10)-(22).

2) Formulation $E_{3}$ minimizes (1), subject to (2)-(5) and (7)-(22).

3) Formulation $E_{4}$ minimizes (1), subject to (2)-(3), (23)-(28), (10)-(15), (30)-(31) and (18)-(22).

4) Formulation $E_{5}$ minimizes (1), subject to constraints (2), (33), (23)-(28), (10), (11), (13)-(15), (30)-(31) and (18)-(22)

\subsection{Valid inequalities}

We make use of four polynomial size valid inequalities. These were used by several authors for variants of LRPs. The first inequalities are special case of classical subtour elimination constraints for the Traveling Salesman Problem (Dantzig et al., 1954) for two nodes as follows:

$$
x_{i j}^{h}+x_{j i}^{h} \leq 1 \quad i, j \in \mathcal{N}_{c}, h \in \mathcal{H} .
$$

Constraints (34) break subtours involving two customers only. The second valid inequality, which was used by Labbé et al. (2004) for plant-cycle location problem is as follows:

$$
z_{i k} \leq y_{k} \quad i \in \mathcal{N}_{c}, k \in \mathcal{N}_{0}
$$


Constraints (35) impose that customer $i$ cannot be assigned to depot $k$ if the latter is not open. We now adapt the next valid inequality described by Achuthan (2003) for the FSMLRPTW as follows:

$$
\sum_{h \in \mathcal{H}} \sum_{k \in \mathcal{N}_{0}} \sum_{i \in \mathcal{N}_{c}} x_{k i}^{h} \geq r_{\alpha}\left(\mathcal{N}_{c}\right)
$$

Constraints (36) provide a lower bound on the number of routes originating from depots where $r_{\alpha}\left(\mathcal{N}_{c}\right)=$ $\left\lceil\sum_{i \in \mathcal{N}_{c}} q_{i} / \max _{h \in \mathcal{H}} Q^{h}\right\rceil$ and $\lceil\bullet\rceil$ is the smallest integer larger than $\bullet$. The fourth and final valid inequality is

$$
\sum_{k \in \mathcal{N}_{0}} y_{k} \geq y_{\min }
$$

Constraints (37) bounds the number of opened depots from below, where $y_{\min }$ is taken as the minimum number of $k$ opened depots satisfying $\sum_{k \in \mathcal{N}_{0}} D^{k} \geq \sum_{i \in \mathcal{N}_{c}} q_{i}$ after the depots have been sorted in nondecreasing order of their capacity.

\section{Description of the Hybrid Evolutionary Search Algorithm}

This section describes the proposed Hybrid Evolutionary Search Algorithm, called HESA, that has been developed to solve the FSMLRPTW. This algorithm builds on several powerful evolutionary based metaheuristic algorithms (see Koç et al., 2014, 2015; Vidal et al., 2014). In this paper, we additionaly introduce the location-heterogeneous adaptive large neighborhood search (L-HALNS) procedure which is used as a main EDUCATION component in the HESA. Furthermore, we develope an INITIALIZATION to create initial solutions and a PARTITION procedure to split offspring solutions into routes. Finally, we propose a new MUTATION procedure to diversify the solutions.

The general structure of the HESA is sketched in Algorithm 1. The initial population is generated by using the INITIALIZATION procedure (line 1). Two parents are selected (line 3) through a binary tournament process, where a crossover operation creates a new offspring $C$ (line 4), which then undergoes the PARTITION procedure (line 5). The EDUCATION procedure uses the L-HALNS operators to educate offspring $C$ and inserts it back into the population (line 6). The probabilities associated with the L-HALNS operators used in the EDUCATION procedure are updated by an Adaptive Weight Adjustment Procedure (AWAP) (line 7). The INTENSIFICATION procedure which is based on the L-HALNS is run on elite solutions (line 8). As new offsprings are added, the population size $n_{a}$ which is limited by $n_{p}+n_{o}$, changes over the iterations. The constant $n_{p}$ denotes the size of the population initialized at the beginning of the algorithm and the constant 
$n_{o}$ is the maximum allowable number of offspring that can be inserted into the population. If the population size $n_{a}$ reaches $n_{p}+n_{o}$ at any iteration, then a survivor selection mechanism is applied (line 9). The MUTATION procedure is applied to a randomly selected individual from the population with probability $p_{m}$ (line 10). When the number $\varpi$ of iterations without improvement in the incumbent solution is reached, the HESA terminates (line 11). For further implementation details on the ALNS based education, parent selection, crossover, AWAP, intensification and survivor selection sections the reader is referred to Koç et al. (2015).

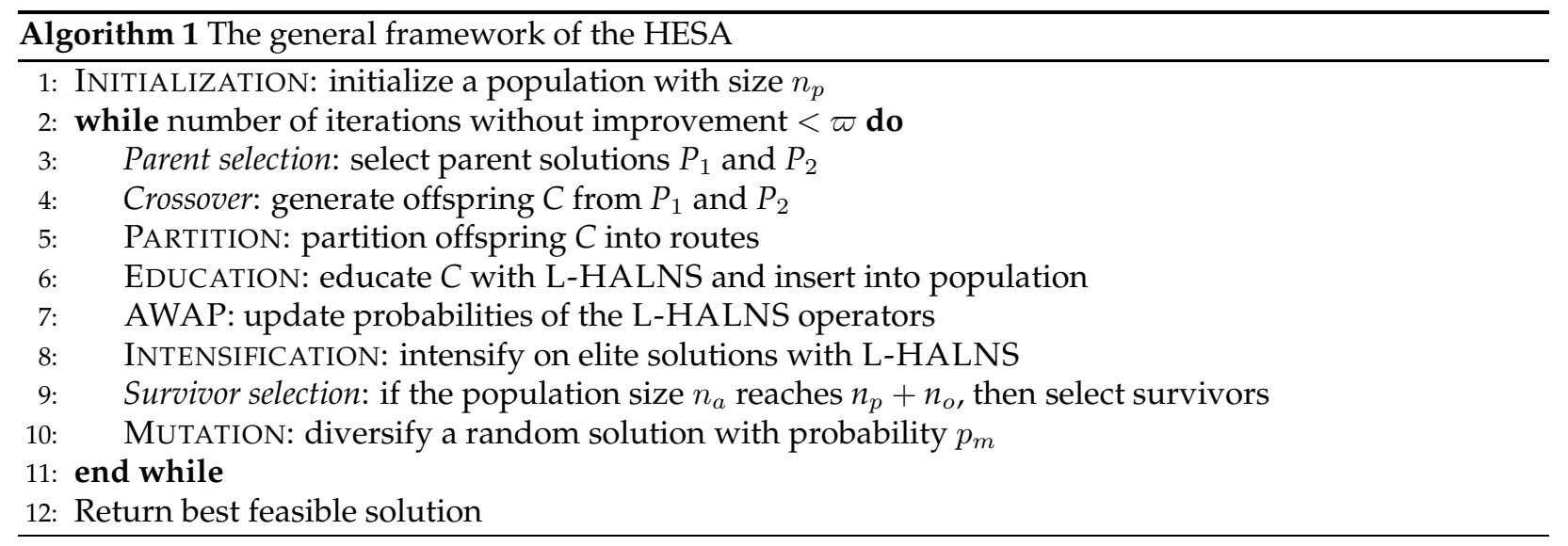

In what follows, we detail the algorithmic features specifically designed for the FSMLRPTW. Section 3.1 presents the INITIALIZATION procedure of the population. The PARTITION procedure is described in Section 3.2. Section 3.3 presents the offspring EDUCATION procedure. Finally, the MUTATION procedure is desribed in Section 3.4 .

\subsection{INITIALIZATION}

We use a three-phase INITIALIZATION procedure to generate an initial population. The first and second phases generate the initial solution while the third phase creates other solutions. In the first phase, customers are assigned to the depots. Initially, the closest depot according to its distance is calculated for each customer, and the customers are listed in non-increasing order of distance. Each customer is then assigned to its closest depot starting from the top of the list, without violating the depot capacities. These steps are applied repeatedly to the residual customers who could not be assigned to their closest depots due to depot capacity constraints. In this case they are assigned to their closest feasible depot. In the second phase, routes are constructed for each depot by applying the Clarke and Wright (1964) algorithm and by selecting the largest vehicle type for each route. In the third phase, new individuals are created by applying the L-HALNS operators to the initial solution until the initial population size reaches $n_{p}$. A removal operator 
is randomly selected from a list of diversification based removal operators and a greedy insertion with a noise function operator is used as an insertion operator (see Section 3.3). Both of these operators are used in order to diversify the initial population. The number of nodes removed is randomly chosen from the initialization interval $\left[b_{l}^{i}, b_{u}^{i}\right]$ in the destroy phase. The interval is defined by a lower and an upper bound calculated as a percentage of the total number of nodes in a given instance.

\subsection{PARTITION}

We introduce a PARTITION procedure to be used in the L-HALNS (see Section 3.3). The objective of this procedure is to split the solution into routes after the parent selection and the crossover phases during the algorithm. Before applying this procedure, each solution is represented as a permutation of customers and can therefore be viewed as a list $L_{r}$ to be used for removals. This procedure includes two phases. In the first phase, all customers of the newly generated solution are put into $L_{r}$. In the second phase, we use an intensification-based insertion operator (greedy insertion operator) to insert the customers of $L_{r}$ to their best possible position. The PARTITION procedure yields a feasible solution for the FSMLRPTW which is inserted into the population.

\subsection{EDUCATION}

At every iteration, the EDUCATION procedure is applied to the newly generated offspring to improve its quality. The ALNS used as a way of educating the solutions, as in Koç et al. (2015), is basically an improvement heuristic. It consists of two procedures: removal, followed by insertion. In the removal procedure, $n^{\prime}$ nodes are iteratively removed by the intensification based removal operators and placed in the removal list $L_{r}$, where $n^{\prime}$ is in the interval $\left[b_{l}^{e}, b_{u}^{e}\right]$ for the destroy operators. In the insertion procedure, the nodes of $L_{r}$ are iteratively inserted into a least-cost position in the incomplete solution.

Here, we introduce the Location-HALNS (L-HALNS) which integrates fleet sizing and the location decisions within the removal and insertion operators. This procedure differs from the Heterogeneous ALNS (HALNS) developed by Koç et al. (2014) to educate the solutions in the context of a heterogeneous fleet because the latter did not account for the location decisions. When a node is removed, we check whether the resulting route can be served by a smaller vehicle, and we also check whether the related depot has any customer already assigned to it. We then update the solution accordingly. If inserting a node requires additional vehicle capacity or requires opening a new depot, we then consider the option of using larger 
vehicles or the option of opening the least cost depot not yet open. More formally, for each node $i \in \mathcal{N}_{0} \backslash L_{r}$, let $f^{h}$ be the vehicle fixed cost and let $g^{k}$ be the depot cost associated with this node. Let $\Delta(i)$ be the distance saving obtained as a result of using a removal operator on node $i$. Let $g_{r}^{k *}$ be the depot fixed cost and let $f_{r}^{h *}$ be the vehicle fixed cost after the removal of node $i$, i.e., $g_{r}^{k *}$ is modified only if node $i$ is the only node of depot $k$ and $f_{r}^{h *}$ is modified only if the route containing node $i$ can be served by a smaller vehicle when removing node $i$. The savings in depot fixed cost and vehicle fixed cost can be expressed as $g^{k}-g_{r}^{k *}$ and $f^{h}-f_{r}^{h *}$, respectively. Thus, for each removal operator, the total savings resulting from removing node $i \in \mathcal{N}_{0} \backslash L_{r}$, denoted $R C(i)$, is calculated as follows:

$$
R C(i)=\Delta(i)+\left(g^{k}-g_{r}^{k *}\right)+\left(f^{h}-f_{r}^{h *}\right) .
$$

In the destroyed solution, the insertion cost of node $j \in L_{r}$ after node $i$ is defined as $\Omega(i, j)$ on a given node $i \in \mathcal{N}_{0} \backslash L_{r}$. Let $g_{a}^{k *}$ be the depot fixed cost and let $f_{a}^{h *}$ be the vehicle fixed cost after the insertion of node $i$, i.e., $g_{r}^{k *}$ is modified only if node $i$ requires to open a new depot, or $f_{r}^{h *}$ is modified only if the route containing node $i$ necessitates the use of a larger capacity vehicle after inserting node $i$. The cost differences in depot fixed cost and vehicle fixed cost can be expressed as $g_{r}^{k *}-g^{k}$ and $f_{a}^{h *}-f^{h}$, respectively. Thus, the total insertion cost of node $i \in \mathcal{N}_{0} \backslash L_{r}$ is $I C(i)$, for each insertion operator is

$$
I C(i)=\Omega(i, j)+\left(g_{r}^{k *}-g^{k}\right)+\left(f_{a}^{h *}-f^{h}\right) .
$$

Figure 1 provides an example of the removal and insertion phases of the L-HALNS procedure.

\subsubsection{Diversification based removal operators}

The first and second diversification based removal operators were applied by Hemmelmayr et al. (2012), and the third one was used by Ropke and Pisinger (2006a). We introduce the fourth one as a new operator specific to the FSMLRPTW.

1. Depot closing removal (DR): This operator randomly selects an open depot and closes it. The operator removes all customers of this depot. The DR operator also randomly selects a closed depot and opens it.

2. Depot opening removal (DOR): The DOR operator randomly opens a closed depot. The $n^{\prime}$ customers removed from the solution are those which are closest to this depot according to travel cost.

3. Random removal (RR): This operator randomly selects $n^{\prime}$ customers and puts them into the removal list. 

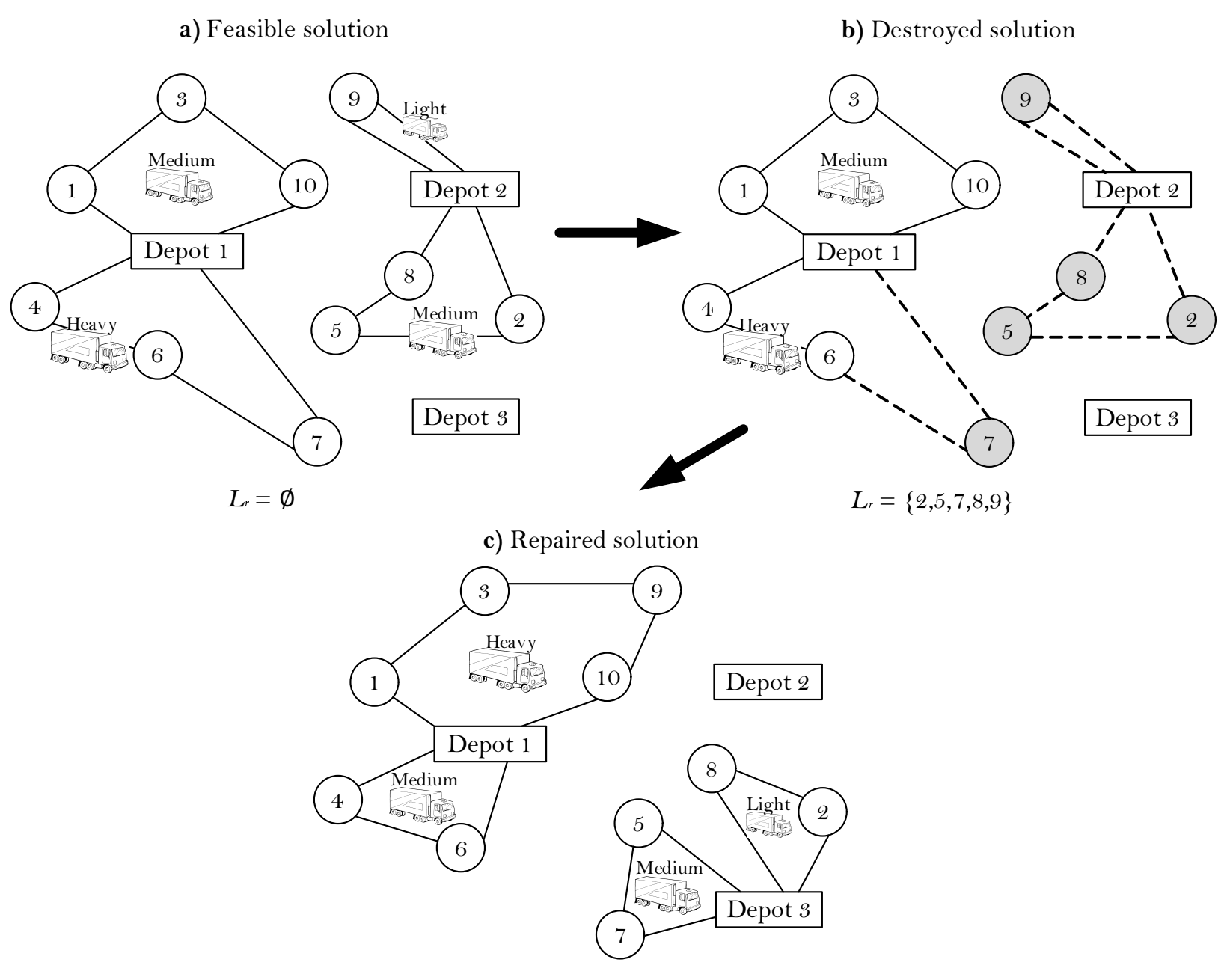

$L_{r}=\varnothing$

Figure 1: Illustration of the L-HALNS procedure 
4. Depot distance removal (DDR): The DSR operator is based on the DR operator but differs from it by the criterion employed to open a closed depot. To open a depot, this operator selects a closest depot with respect to a removed one.

\subsubsection{Intensification based removal operators}

We now present the eight intensification based removal operators used in our algorithm. The first seven were used in several papers (Ropke and Pisinger, 2006a,b; Demir et al., 2012; Koç et al., 2015), whereas the last one is new.

1. Neighborhood removal (NR): The general idea behind the NR operator is to remove $n^{\prime}$ customers from routes that are extreme with respect to the average distance of a route.

2. Worst distance removal (WDR): The WDR operator iteratively removes $n^{\prime}$ high cost customers, where the cost is based on the distance.

3. Worst time removal (WTR): This operator is a variant of the WDR operator. For each node, costs are calculated, depending on the deviation between the arrival time and the beginning of the time window. The WTR operator iteratively removes $n^{\prime}$ customer from the solution which has the largest deviation.

4. Shaw removal (SR): The SR operator aims to remove a set of $n^{\prime}$ similar customers. The similarity between two customers is based on distance, time, proximity and demands.

5. Proximity-based removal (PBR): This operator is a special case of SR which is solely based on distance.

6. Time-based removal (TBR): The TBR operator is another variant of SR. The selection criterion of a set of customers is solely based on time.

7. Demand based-removal (DBR): The DBR operator is yet another variant of SR which is solely based on demand.

8. Depot closing removal (DCR): The aim of the DCR operator is to calculate the utilization efficiency of each depot $\Phi(k)\left(k \in \mathcal{N}_{0}\right)$ and remove from solution the one with the least $\Phi(k)$ value. $\Phi(k)$ is expressed as the ratio of the total demand of depot $k$ over the capacity of depot $k$ :

$$
\Phi(k)=\frac{\sum_{i \in \mathcal{N}_{c}} q_{i} z_{i k}}{D^{k}} .
$$




\subsubsection{Insertion operators}

Two insertion operators (Ropke and Pisinger, 2006a,b; Demir et al., 2012; Koç et al., 2015) are used in the repair phase of the EDUCATION procedure.

1. Greedy insertion operator (GIO): This operator finds the best possible insertion position for all nodes in $L_{r}$ while the cost calculation is based on distance. The insertion process is iteratively applied to all nodes in $L_{r}$.

2. Greedy insertion with noise function operator (GINFO): The GINFO operator is a variant of the GIO operator that extends it by allowing a degree of freedom in selecting the best possible insertion position for a node.

\subsection{MUtATiON}

We introduce a MUTATION procedure to increase the population diversity over the iterations (e.g., Nagata et al., 2010). This procedure is applied with probability $p_{m}$. An individual $C$ different from the elite individuals is randomly selected. A randomly selected diversification based removal operator and the GINFO operator are then used in order to diversify the selected individual $C$. These two operators are applied to change the positions of a specific number of nodes, which are chosen from the interval $\left[b_{l}^{m}, b_{u}^{m}\right]$ of removable nodes.

\section{Computational experiments}

In this section, we present the results of computational experiments performed in order to assess the performance of the formulations and the HESA. All experiments were conducted on a server with one gigabyte RAM and Intel Xeon $2.6 \mathrm{GHz}$ processor. We used CPLEX 12.5 with its default settings as the optimizer to solve the integer programming formulations. The HESA was implemented in $\mathrm{C}++$.

Section 4.1 describes the benchmark instances and the experimental settings. The aim of the computational experiments is threefold: (i) to analyze the effect of the metaheuristic components (Section 4.2), (ii) to evaluate the formulation in terms of solving the FSMLRPTW to optimality on small-size instances (Section 4.3), and (iii) to compare and test the integer programming formulation which is integrated with valid inequalities and the HESA on small-medium-and large-size instances (Section 4.4). 


\subsection{Benchmark instances}

Benchmark data sets for the FSMLRPTW were generated by considering the data set described by Liu and Shen (1999) for the Fleet Size and Mix VRP with Time Windows (FSMVRPTW) and derived from the classical Solomon (1987) VRP with Time Windows instances with 100 nodes. These sets include 56 instances, split into a random data set $R$, a clustered data set $C$ and a semi-clustered data set $R C$. The sets $\mathrm{R} 1, \mathrm{C} 1$ and $\mathrm{RC} 1$ have a short scheduling horizon and small vehicle capacities, in contrast to the sets R2, C2 and RC2 which have a longer scheduling horizon and larger vehicle capacities. Liu and Shen (1999) also introduced three cost structures, namely A, B and C, and several vehicle types with different capacities and fixed vehicle costs for each of the 56 instances. In our data sets, we have used the cost structure A and generated small-size (10-15-20-25-30-customer) as well as medium and large-size (50-75-100-customer) instances. We have selected the first customers of each data sets to generate the instances, i.e., the first 10 customers, 15 customers, and so on, of each Liu and Shen instance.

We followed a procedure similar to that used for the LRP benchmark instances (see Barreto, 2004; AlbaredaSambola et al., 2005; Prodhon, 2006; Karaoglan et al., 2012) to generate our depot characteristics. To this end, new depots features were added to each instance of Liu and Shen, as shown in Appendix A.1. The depot characteristics were generated using discrete uniform distributions. The depot coordinates are in the range $[0,100]^{2}$. For the sets $\mathrm{C} 1, \mathrm{C} 2, \mathrm{R} 1, \mathrm{R} 2, \mathrm{RC} 1$ and RC2, the depot costs are in the range [38000, 50000], [90000, 120000], [17000, 25000], [85000, 100000], [17000, 26000] and [85000, 100000], respectively. The intervals for depot capacities for each data set and for small-, medium- and large-size instances are given in Table 1. However, these data ensure the opening of at least two depots for each instance. In the original Solomon instances there is only one depot; we have used this depot's time windows for other depots as well, i.e., in each data set, time windows are same for all depots.

Table 1: The intervals for depot capacities

\begin{tabular}{|l|llllll|}
\hline$\left|\mathcal{N}_{c}\right|$ & Data set & \multicolumn{5}{l|}{} \\
\cline { 2 - 7 } & $\mathrm{C} 1$ & $\mathrm{C} 2$ & $\mathrm{R} 1$ & $\mathrm{R} 2$ & $\mathrm{RC} 1$ & $\mathrm{RC} 2$ \\
\hline 10 & {$[90,110]$} & {$[100,120]$} & {$[80,85]$} & {$[90,105]$} & {$[150,180]$} & {$[160,190]$} \\
15 & {$[150,210]$} & {$[160,220]$} & {$[110,160]$} & {$[120,180]$} & {$[210,250]$} & {$[230,270]$} \\
20 & {$[190,220]$} & {$[210,240]$} & {$[140,170]$} & {$[150,190]$} & {$[220,260]$} & {$[270,320]$} \\
25 & {$[220,320]$} & {$[230,340]$} & {$[270,310]$} & {$[290,305]$} & {$[290,340]$} & {$[360,420]$} \\
30 & {$[360,410]$} & {$[360,430]$} & {$[270,310]$} & {$[280,340]$} & {$[370,430]$} & {$[370,430]$} \\
50 & {$[540,610]$} & {$[580,660]$} & {$[390,510]$} & {$[450,530]$} & {$[630,710]$} & {$[670,740]$} \\
75 & {$[810,900]$} & {$[830,930]$} & {$[550,760]$} & {$[630,790]$} & {$[730,860]$} & {$[760,900]$} \\
100 & {$[780,1000]$} & {$[800,1030]$} & {$[720,1000]$} & {$[700,1100]$} & {$[790,1180]$} & {$[780,1300]$} \\
\hline
\end{tabular}

Evolutionary algorithms use a set of correlated parameters. All algorithmic parametric values were set as in Koç et al. (2015), where an extensive meta-calibration procedure was used to generate effective parameter values for the FSMVRPTW. These authors initially used the parameters suggested by Vidal et al. (2014) 
for the genetic algorithm, and by Demir et al. (2012) for the ALNS. The authors then conducted several experiments to further fine tune the parameters on randomly selected instances.

\subsection{Sensitivity analysis of method components}

This section compares four versions of the HESA, the details of which can be found in Table 2. We present four sets of experiments on randomly selected 100-customer instances; C101, C203, R101, R211, RC105 and RC207.

Table 2: Sensitivity analysis experiment setup

\begin{tabular}{|l|lll|}
\hline Version & EDUCATION & INTENSIFICATION & MUTATION \\
\hline$(1)$ & No & No & No \\
$(2)$ & Yes & No & No \\
$(3)$ & Yes & Yes & No \\
HESA & Yes & Yes & Yes \\
\hline
\end{tabular}

Table 3 presents the best results of ten runs for each of four versions. The columns display the instance type, the total cost, percentage deterioration in solution quality (Dev) of the three versions with respect to the HESA, and the computation time in seconds (Time). The row named Avg shows the average results. These results clearly indicate the benefit of including the EDUCATION, INTENSIFICATION and MUTATION procedures within the HESA. The HESA is consistently superior to all other versions on all instances. Version (1) performs worse than all other three versions. The superiority of version (3) over version (2) confirms the usefulness of the INTENSIFICATION procedure in the algorithm. The computation times for all versions are of similar magnitude.

Table 3: Sensitivity analysis of the HESA components

\begin{tabular}{|l|lll|llll|lll|ll|}
\hline \multirow{2}{*}{ Instance } & Version (1) & & \multicolumn{3}{|l|}{ Version (2) } & Version (3) & & HESA & \\
\cline { 2 - 11 } & Total cost & Dev & Time & Total cost & Dev & Time & Total cost & Dev & Time & Total cost & Time \\
\hline C101 & 89091.74 & 4.37 & 274.19 & 87091.74 & 2.17 & 279.34 & 86590.81 & 1.61 & 286.38 & 85199.09 & 297.32 \\
C203 & 199501.19 & 4.33 & 286.24 & 196801.71 & 3.02 & 294.13 & 192841.36 & 1.03 & 301.14 & 190864.00 & 308.09 \\
R101 & 43941.23 & 4.91 & 271.39 & 42841.17 & 2.47 & 276.41 & 42640.26 & 2.01 & 282.51 & 41782.20 & 292.25 \\
R211 & 185112.41 & 5.44 & 235.29 & 180119.73 & 2.81 & 240.13 & 178152.37 & 1.74 & 244.09 & 175051.00 & 247.03 \\
RC105 & 41640.21 & 3.96 & 249.13 & 41340.21 & 3.27 & 271.31 & 40840.17 & 2.08 & 276.91 & 39990.10 & 281.31 \\
RC207 & 183071.91 & 4.26 & 280.17 & 179270.13 & 2.23 & 290.43 & 178274.36 & 1.68 & 294.37 & 175280.00 & 300.42 \\
& & & & & & & & & & & \\
Avg & & 4.54 & 266.07 & & 2.66 & 275.29 & & 1.69 & 280.90 & & 287.74 \\
\hline
\end{tabular}

\subsection{Performance of the formulations}

The formulations $E_{1}, E_{2}, E_{3}, E_{4}$ and $E_{5}$ are examined in terms of their ability to solve the FSMLRPTW to optimality on small-size (20-, 25-, and 30-customer) instances. To analyze the computational results, 
we used the following performance measures: the deviation (Dev) and computation time in seconds (Time) averaged over all instances for each instance set (over a total of 840 experiments), and the number of optimal solutions (\#Op) obtained within one hour of computation time. Dev is the percentage deviation between the Upper Bound (UB) and the best-known Lower Bound (LB), i.e., 100 (UB - LB)/ UB. The upper bound is the optimal or best known solution obtained by solving the formulations.

Table 4 presents comparative average results over the five formulations. The first column displays the instance sets, and the following two columns show the number of customers $\left|\mathcal{N}_{c}\right|$ and the number of depots $\left|\mathcal{N}_{0}\right|$, respectively. The overall results of $E_{1}$ are better than those of $E_{3}$, thus we infer that constraints (6) strengthen formulation $E_{1}$. The results shown in Table 4 indicate that formulation $E_{4}$ performs better than the other models in terms of reaching optimal solutions within one hour of computation time. These results imply that instead of constraints (4)-(9), using constraints (23)-(28) which include the aggregated variables $f_{i j}$, and instead of constraints (16)-(17) using constraints (30)-(31), which include the aggregated variables $f_{i j}$, yields better formulations for the FSMLRPTW. Formulation $E_{4}$ yields 27 optimal solutions out of 56 instances for the 20-customer instances, 13 out of 56 instances for 25-customer instances, and nine out of 56 instances for 30-customer instances within the given time limit of one hour. In terms of computation time, $E_{4}$ provides on average lower computation time than the other formulations.

Table 4: Average results of the formulations

\begin{tabular}{|c|c|c|c|c|c|c|c|c|c|c|c|c|c|c|c|c|c|}
\hline \multirow[t]{2}{*}{ Instance set } & \multirow[t]{2}{*}{|| $\mathcal{N}_{c} \mid$} & \multirow[t]{2}{*}{|| $\mathcal{N}_{0} \mid$} & \multicolumn{3}{|l|}{$E_{1}$} & \multicolumn{3}{|l|}{$\overline{E_{2}}$} & \multicolumn{3}{|l|}{$E_{3}$} & \multicolumn{3}{|l|}{$E_{4}$} & \multicolumn{3}{|l|}{$E_{5}$} \\
\hline & & & Dev & Time & $\# O p$ & Dev & Time & $\# O p$ & Dev & Time & $\# O p$ & Dev & Time & $\# O p$ & Dev & Time & $\# \mathrm{O} p$ \\
\hline C1 (9) & 20 & 5 & 0.07 & 2245.17 & 4 & 0.07 & 1978.42 & 5 & 0.08 & 2417.07 & 5 & 0.08 & 2505.74 & 4 & 0.08 & 2467.02 & 3 \\
\hline C2 (8) & 20 & 5 & 0.07 & 2245.17 & 7 & 0.00 & 844.86 & 7 & 0.42 & 2782.58 & 2 & 0.00 & 573.49 & 8 & 0.00 & 359.59 & 8 \\
\hline R1 (12) & 20 & 5 & 0.29 & 2947.26 & 3 & 0.28 & 2936.22 & 3 & 0.32 & 3019.03 & 2 & 0.21 & 3066.77 & 3 & 0.20 & 3019.31 & 2 \\
\hline R2 (11) & 20 & 5 & 0.01 & 2059.15 & 7 & 0.01 & 1975.49 & 7 & 0.27 & 3291.85 & 1 & 0.00 & 751.98 & 11 & 0.00 & 894.84 & 10 \\
\hline RC1 (8) & 20 & 5 & 0.17 & 3228.86 & 1 & 0.18 & 3251.18 & 1 & 0.25 & 3208.96 & 1 & 0.17 & 3222.55 & 1 & 0.17 & 3192.03 & 1 \\
\hline RC2 (8) & 20 & 5 & 0.07 & 3600.00 & 0 & 0.06 & 3600.00 & 0 & 0.07 & 3600.00 & 0 & 0.07 & 3600.00 & 0 & 0.06 & 3600.00 & 0 \\
\hline Avg (Total) & & & 0.11 & 2720.94 & (22) & 0.10 & 2431.03 & (23) & 0.23 & 3053.25 & (11) & 0.09 & 2286.76 & (27) & 0.09 & 2255.46 & (24) \\
\hline C1 (9) & 25 & 5 & 0.12 & 2669.77 & 3 & 0.11 & 2480.23 & 3 & 0.16 & 3600.00 & 0 & 0.14 & 3207.57 & 1 & 0.14 & 3432.45 & 1 \\
\hline C2 (8) & 25 & 5 & 0.02 & 1607.91 & 5 & 0.05 & 1932.03 & 5 & 0.31 & 3151.28 & 1 & 0.00 & 1215.17 & 6 & 0.00 & 1120.62 & 6 \\
\hline R1 (12) & 25 & 5 & 0.44 & 3302.73 & 1 & 0.43 & 3302.31 & 1 & 0.32 & 3151.80 & 2 & 0.35 & 3300.77 & 1 & 0.37 & 3301.73 & 1 \\
\hline R2 (11) & 25 & 5 & 0.17 & 3325.51 & 1 & 0.11 & 3057.42 & 2 & 0.24 & 3308.48 & 1 & 0.03 & 2597.39 & 4 & 0.06 & 2957.59 & 2 \\
\hline RC1 (8) & 25 & 5 & 0.22 & 3600.00 & 0 & 0.22 & 3289.92 & 1 & 0.23 & 3305.74 & 1 & 0.20 & 3204.17 & 1 & 0.21 & 3367.11 & 1 \\
\hline RC2 (8) & 25 & 5 & 0.10 & 3600.00 & 0 & 0.07 & 3600.00 & 0 & 0.10 & 3600.00 & 0 & 0.09 & 3600.00 & 0 & 0.07 & 3600.00 & 0 \\
\hline Avg (Total) & & & 0.18 & 3017.65 & (10) & 0.16 & 2943.65 & (12) & 0.23 & 3352.88 & (5) & 0.14 & 2854.18 & (13) & 0.14 & 2963.25 & (11) \\
\hline C1 (9) & 30 & 5 & 0.32 & 3600.00 & 0 & 0.26 & 3264.60 & 1 & 0.32 & 3600.00 & 0 & 0.32 & 3600.00 & 0 & 0.33 & 3600.00 & 0 \\
\hline C2 (8) & 30 & 5 & 0.02 & 2276.50 & 5 & 0.00 & 1478.03 & 6 & 0.25 & 2975.67 & 2 & 0.10 & 1545.67 & 6 & 0.12 & 2083.98 & 5 \\
\hline R1 (12) & 30 & 5 & 0.48 & 3305.80 & 1 & 0.50 & 3317.96 & 1 & 0.72 & 3310.17 & 1 & 0.49 & 3303.81 & 1 & 0.47 & 3303.81 & 1 \\
\hline R2 (11) & 30 & 5 & 0.21 & 3600.00 & 0 & 0.14 & 3434.65 & 1 & 0.33 & 3600.00 & 0 & 0.08 & 3159.86 & 2 & 0.07 & 3087.20 & 2 \\
\hline RC1 (8) & 30 & 5 & 0.32 & 3600.00 & 0 & 0.28 & 3600.00 & 0 & 0.34 & 3600.00 & 0 & 0.38 & 3600.00 & 0 & 0.32 & 3600.00 & 0 \\
\hline RC2 (8) & 30 & 5 & 1.26 & 3600.00 & 0 & 0.75 & 3600.00 & 0 & 1.01 & 3600.00 & 0 & 0.8 & 3600.00 & 0 & 0.67 & 3600.00 & 0 \\
\hline Avg (Total) & & & 0.43 & 3330.38 & (6) & 0.32 & 3115.87 & (9) & 0.49 & 3447.64 & (3) & 0.36 & 3134.89 & (9) & 0.33 & 3212.50 & (8) \\
\hline
\end{tabular}

We now investigate the effect of the valid inequalities on formulation $E_{4}$. To this end, we have conducted experiments on instances of the same size and we have used the same performance measures as shown in 
Table 5: Effect of the valid inequalities

\begin{tabular}{|c|c|c|c|c|c|c|c|c|c|c|c|c|c|c|}
\hline \multirow[t]{2}{*}{ Instance set } & \multirow[t]{2}{*}{$\overline{\left|\mathcal{N}_{c}\right|}$} & \multirow[t]{2}{*}{$\mid \overline{\mathcal{N}_{0} \mid}$} & \multicolumn{3}{|l|}{$\overline{V_{1}}$} & \multicolumn{3}{|l|}{$\overline{V_{2}}$} & \multicolumn{3}{|l|}{$V_{3}$} & \multicolumn{3}{|l|}{$\overline{V_{4}}$} \\
\hline & & & $\overline{\text { Dev }}$ & Time & $\# O p$ & $\overline{\text { Dev }}$ & Time & $\# O p$ & $\overline{\mathrm{Dev}}$ & Time & $\# O p$ & $\overline{\text { Dev }}$ & Time & $\# O p$ \\
\hline C1 (9) & 20 & 5 & 0.07 & 1728.73 & 5 & 0.08 & 1694.42 & 5 & 0.06 & 1705.47 & 5 & 0.07 & 1881.50 & 5 \\
\hline C2 (8) & 20 & 5 & 0.07 & 2245.17 & 7 & 0.01 & 714.34 & 7 & 0.01 & 628.12 & 7 & 0.00 & 615.45 & 8 \\
\hline R1 (12) & 20 & 5 & 0.21 & 3007.92 & 2 & 0.24 & 2757.75 & 3 & 0.24 & 2755.51 & 3 & 0.20 & 2613.15 & 3 \\
\hline R2 (11) & 20 & 5 & 0.01 & 1109.64 & 9 & 0.01 & 1141.27 & 9 & 0.01 & 1059.42 & 10 & 0.01 & 1029.43 & 10 \\
\hline RC1 (8) & 20 & 5 & 0.17 & 3224.54 & 1 & 0.17 & 3208.28 & 1 & 0.17 & 3236.80 & 1 & 0.16 & 3199.37 & 1 \\
\hline RC2 (8) & 20 & 5 & 0.07 & 3600.00 & 0 & 0.08 & 3600.00 & 0 & 0.08 & 3600.00 & 0 & 0.07 & 3600.00 & 0 \\
\hline Avg (Total) & & & 0.10 & 2486.00 & 24 & 0.10 & 2186.01 & 25 & 0.10 & 2164.22 & 26 & 0.09 & 2156.48 & 27 \\
\hline C1 (9) & 25 & 5 & 0.13 & 2810.01 & 2 & 0.13 & 2795.28 & 2 & 0.12 & 2795.30 & 2 & 0.10 & 3193.31 & 1 \\
\hline C2 (8) & 25 & 5 & 0.01 & 1283.90 & 6 & 0.01 & 1369.76 & 6 & 0.01 & 1308.49 & 6 & 0.01 & 1126.88 & 6 \\
\hline R1 (12) & 25 & 5 & 0.31 & 3058.63 & 2 & 0.20 & 3143.78 & 2 & 0.20 & 3074.96 & 2 & 0.20 & 3075.17 & 2 \\
\hline $\mathrm{R} 2$ (22) & 25 & 5 & 0.06 & 2715.95 & 2 & 0.10 & 3561.73 & 2 & 0.25 & 3571.71 & 2 & 0.04 & 2839.60 & 3 \\
\hline RC1 (8) & 25 & 5 & 0.22 & 3283.27 & 1 & 0.20 & 3289.40 & 1 & 0.10 & 3301.02 & 1 & 0.30 & 3258.57 & 1 \\
\hline RC2 (8) & 25 & 5 & 0.10 & 3600.00 & 0 & 0.14 & 3600.00 & 0 & 0.10 & 3600.00 & 0 & 0.07 & 3600.00 & 0 \\
\hline Avg (Total) & & & 0.14 & 2791.96 & 13 & 0.13 & 2959.99 & 13 & 0.13 & 2941.91 & 13 & 0.12 & 2848.92 & 13 \\
\hline C1 (9) & 30 & 5 & 0.28 & 3197.45 & 1 & 0.30 & 3591.70 & 0 & 0.32 & 3591.61 & 1 & 0.28 & 3249.88 & 1 \\
\hline C2 (8) & 30 & 5 & 0.14 & 2081.03 & 4 & 0.14 & 1822.59 & 5 & 0.17 & 2180.70 & 4 & 0.19 & 1930.52 & 5 \\
\hline R1 (12) & 30 & 5 & 0.43 & 3296.05 & 1 & 0.50 & 3295.51 & 1 & 0.41 & 3295.57 & 1 & 0.40 & 3295.65 & 1 \\
\hline $\mathrm{R} 2$ (22) & 30 & 5 & 0.10 & 3356.11 & 1 & 0.11 & 3434.18 & 1 & 0.06 & 3399.88 & 1 & 0.06 & 3321.14 & 2 \\
\hline RC1 (8) & 30 & 5 & 0.28 & 3600.00 & 0 & 0.35 & 3600.00 & 0 & 0.34 & 3600.00 & 0 & 0.31 & 3600.00 & 0 \\
\hline RC2 (8) & 30 & 5 & 0.89 & 3600.00 & 0 & 0.65 & 3600.00 & 0 & 0.70 & 3600.00 & 0 & 0.67 & 3600.00 & 0 \\
\hline Avg (Total) & & & 0.35 & 3188.44 & 7 & 0.34 & 3224.00 & 7 & 0.33 & 3277.96 & 7 & 0.32 & 3066.20 & 9 \\
\hline
\end{tabular}

Table 4. We used four variations of $E_{4}$ denoted as $V_{1}, V_{2}, V_{3}$ and $V_{4}$. Variation $V_{1}$ is formulation $E_{4}$ with valid inequalities (34), $V_{2}$ is formulation $E_{4}$ with valid inequalities (34) and (35), $V_{3}$ is formulation $E_{4}$ with valid inequalities (34)-(36), and $V_{4}$ is formulation $E_{4}$ with valid inequalities (34)-(37). Table 5 summarizes the average results of the effect of the valid inequalities on formulation $E_{4}$. These results show that $V_{4}$, which includes all valid inequalities, performs better than all other variations in terms of reaching optimal solutions within one hour of computation time. For the 20-, 25-, and 30-customer instances, the solution times are 2286.76, 2854.18 and 3134.89 seconds for $E_{4}$, but these times are 2156.48, 2848.92, 3066.20 seconds for $V_{4}$. Our results indicate that $V_{4}$ yields same number of optimal solutions as $E_{4}$, but within smaller computation times.

\subsection{Comparative Analysis}

We now present a comparative analysis of the results of the HESA and of the formulation $E_{4}$ integrated with valid inequalities, denoted by $E_{4}^{v}$. Each instance was solved once with the HESA, and once with $E_{4}^{v}$. For $E_{4}^{v}$, a common time limit of three hours was imposed on the solution time for all instances. For the HESA, ten separate runs are performed for each instance, the best one of which is reported.

Tables 6 and 7 summarize the average results of the HESA compared with $E_{4}^{v}$. For detailed results, the reader is referred to Appendix A. In Tables 6 and 7, the first column displays the LP relaxation value of $E_{4}^{v}$, 
obtained by relaxing the integrality constraints (18) on the $X_{i j}^{h}$ variables only. Such a partial LP relaxation provides better quality lower bounds compared to a full relaxation where the integer restrictions (18)-(20) on all binary variables are relaxed, and in comparable solution times. The remaining columns show the total time of all 10 runs of the HESA (Total time (s.)), the time of the best solution (Time (s.)), the percent deviation values of the total cost $\left(\operatorname{Dev}_{T C}\right)$ and of the vehicle cost $\left(\operatorname{Dev}_{V C}\right)$ found by $E_{4}^{v}$ with respect to the HESA. In the Time column, “*” denotes that the instance was not solved to optimality within three hours.

Table 6 shows that the HESA finds almost the same solutions as those of $E_{4}^{v}$ but in a substantially smaller amount of time on the small-size FSMLPRTW instances. The average time required by $E_{4}^{v}$ to solve 10-, 15-, 20-, 25- and 30-customer instances to optimality are 26.26, 3234.61, 6012.79, 8056.69 and 9359.53 seconds, respectively. For the HESA, the respective statistics are 3.18, 3.95, 6.58, 8.93 and 13.05 seconds.

As can be observed from Table 7, the results clearly indicate that the HESA runs quickly, also for medium and large-size instances. In particular, the algorithm requires 90.08, 167.82 and 299.98 seconds of average computation time to solve 50-, 75- and 100-customer instances, respectively. The HESA is able to produce considerably better results than $E_{4}^{v}$ does in three hours. The improvements in solution values can be as high as $6.42 \%$, with an average of $1.41 \%$ for the 50 -customer instances. Similarly, the average improvement is $5.11 \%$ for the 75 -customer instances, the highest value sitting at $23.09 \%$. The results are even more striking for the 100-customer instances where the average total cost reduction obtained was $19.93 \%$ compared to $E_{4}^{v}$. In case of 50-, 75- and 100-customer instances $E_{4}^{v}$ was not able to find optimal solutions for 166 instances out of 168 within three hours.

We now investigate the effect of the fleet mix composition on the FSMLRPTW instances using the vehicle costs and $\operatorname{Dev}_{V C}$ values in Table 7. The results show that when optimality is guaranteed by $E_{4}^{v}$, the HESA is able to find the optimal fleet mix composition. For the 50- and 75-customer instances, the HESA decreased the vehicle costs by $8.08 \%$ and $32.10 \%$ on average compared to $E_{4}^{v}$. This decrease ranges from 0.00 to $18.68 \%$ and from $4.20 \%$ to $60.60 \%$ for the 50 - and 75 -customer instances, respectively. As for the total costs, the results are even more striking for the 100-customer instances where the average vehicle costs reduction obtained was $53.82 \%$ compared to $E_{4}^{v}$. These results imply that for medium- and large-size instances, the HESA is able to produce substantially lower vehicle costs than $E_{4}^{v}$. 
Table 6: Average results on small-size instances

\begin{tabular}{|c|c|c|c|c|c|c|c|c|c|c|c|c|}
\hline \multirow[t]{2}{*}{ Instance set } & \multirow[t]{2}{*}{$\left|\mathcal{N}_{c}\right|$} & \multirow[t]{2}{*}{$\left|\mathcal{N}_{0}\right|$} & \multicolumn{4}{|l|}{$E_{4}^{v}$} & \multicolumn{6}{|l|}{ HESA } \\
\hline & & & LP relaxation & Vehicle cost & Total cost & Time (s.) & Vehicle cost & Total cost & Total time (s.) & Time (s.) & $\operatorname{Dev}_{T C}$ & $\operatorname{Dev}_{V C}$ \\
\hline C1 (9) & 10 & 3 & 82504.85 & 600.00 & 82674.64 & 0.38 & 600.00 & 82674.64 & 37.83 & 3.57 & 0.00 & 0.00 \\
\hline C2 (8) & 10 & 3 & 191118.19 & 755.56 & 192201.84 & 2.07 & 755.56 & 192201.84 & 34.45 & 3.31 & 0.00 & 0.00 \\
\hline R1 (12) & 10 & 3 & 39382.04 & 911.11 & 39466.34 & 128.64 & 911.11 & 39466.34 & 31.02 & 3.01 & 0.00 & 0.00 \\
\hline R2 (11) & 10 & 3 & 175618.55 & 1066.67 & 176157.95 & 0.96 & 1066.67 & 176157.95 & 34.04 & 3.18 & 0.00 & 0.00 \\
\hline RC1 (8) & 10 & 3 & 35614.85 & 1222.22 & 35716.90 & 10.77 & 1222.22 & 35716.90 & 34.41 & 3.19 & 0.00 & 0.00 \\
\hline RC2 (8) & 10 & 3 & 173434.98 & 1377.78 & 173622.24 & 14.76 & 1377.78 & 173622.24 & 30.75 & 2.82 & 0.00 & 0.00 \\
\hline Avg & & & & & & 26.26 & & & 33.75 & 3.18 & 0.00 & 0.00 \\
\hline C1 (9) & 15 & 4 & 81889.59 & 900.00 & 82059.92 & 5.51 & 900.00 & 82059.92 & 45.83 & 4.51 & 0.00 & 0.00 \\
\hline C2 (8) & 15 & 4 & 186126.81 & 2000.00 & 187193.63 & 9.52 & 2000.00 & 187193.63 & 37.88 & 3.72 & 0.00 & 0.00 \\
\hline R1 (12) & 15 & 4 & 39590.64 & 360.00 & 39702.34 & 6328.34 & 360.00 & 39702.41 & 39.27 & 3.86 & 0.00 & 0.00 \\
\hline R2 (11) & 15 & 4 & 174662.67 & 900.00 & 175190.12 & 98.72 & 900.00 & 175190.12 & 39.53 & 3.88 & 0.00 & 0.00 \\
\hline RC1 (8) & 15 & 4 & 35843.17 & 660.00 & 35961.05 & 3469.07 & 660.00 & 35961.05 & 36.70 & 3.60 & 0.00 & 0.00 \\
\hline RC2 (8) & 15 & 4 & 173626.06 & 650.00 & 173826.21 & 9496.51 & 650.00 & 173826.21 & 42.24 & 4.15 & 0.00 & 0.00 \\
\hline Avg & & & & & & 3234.61 & & & 40.24 & 3.95 & 0.00 & 0.00 \\
\hline C1 (9) & 20 & 5 & 82236.26 & 1200.00 & 82435.86 & 5263.90 & 1200.00 & 82450.45 & 63.52 & 6.25 & 0.02 & 0.00 \\
\hline C2 (8) & 20 & 5 & 186141.23 & 2000.00 & 187224.06 & 601.82 & 2000.00 & 187224.06 & 66.28 & 6.46 & 0.00 & 0.00 \\
\hline R1 (12) & 20 & 5 & 37782.90 & 479.17 & 38139.76 & 8220.94 & 479.17 & 38139.37 & 63.51 & 6.18 & 0.00 & 0.00 \\
\hline R2 (11) & 20 & 5 & 174699.40 & 900.00 & 175239.55 & 1682.87 & 900.00 & 175238.39 & 64.92 & 6.34 & 0.00 & 0.00 \\
\hline RC1 (8) & 20 & 5 & 36075.96 & 870.00 & 36242.45 & 9507.20 & 870.00 & 36246.40 & 71.50 & 7.04 & 0.01 & 0.00 \\
\hline RC2 (8) & 20 & 5 & 173864.43 & 800.00 & 174079.45 & $10800.00^{*}$ & 800.00 & 174078.67 & 74.01 & 7.20 & 0.00 & 0.00 \\
\hline Avg & & & & & & 6012.79 & & & 67.29 & 6.58 & 0.00 & 0.00 \\
\hline C1 (9) & 25 & 5 & 82556.95 & 1500.00 & 82767.35 & 8404.21 & 1500.00 & 82782.57 & 95.31 & 9.33 & 0.02 & 0.00 \\
\hline C2 (8) & 25 & 5 & 186314.82 & 2000.00 & 187234.75 & 2919.09 & 2000.00 & 187234.75 & 87.54 & 8.45 & 0.00 & 0.00 \\
\hline R1 (12) & 25 & 5 & 37930.66 & 613.33 & 38306.32 & 9081.98 & 613.33 & 38287.22 & 94.04 & 9.00 & -0.05 & 0.00 \\
\hline R2 (11) & 25 & 5 & 174795.80 & 900.00 & 175313.88 & 7563.09 & 900.00 & 175311.64 & 90.60 & 8.96 & 0.00 & 0.00 \\
\hline RC1 (8) & 25 & 5 & 36252.02 & 990.00 & 36414.34 & 9571.79 & 990.00 & 36430.18 & 95.26 & 9.03 & 0.04 & 0.00 \\
\hline RC2 (8) & 25 & 5 & 174075.96 & 1000.00 & 174301.53 & $10800.00^{*}$ & 1000.00 & 174300.67 & 91.18 & 8.82 & 0.00 & 0.00 \\
\hline Avg & & & & & & 8056.69 & & & 92.32 & 8.93 & 0.00 & 0.00 \\
\hline C1 (9) & 30 & 5 & 82758.85 & 1800.00 & 83096.43 & 9656.17 & 1800.00 & 83115.24 & 143.41 & 13.84 & 0.02 & 0.00 \\
\hline C2 (8) & 30 & 5 & 186488.20 & 2000.00 & 187253.95 & 5519.71 & 2000.00 & 187252.10 & 128.64 & 12.56 & 0.00 & 0.00 \\
\hline R1 (12) & 30 & 5 & 38126.33 & 764.17 & 38543.11 & 9902.81 & 764.17 & 38495.67 & 119.46 & 11.75 & -0.12 & 0.00 \\
\hline R2 (11) & 30 & 5 & 174930.96 & 900.00 & 175371.60 & 9478.50 & 900.00 & 175356.43 & 147.07 & 14.31 & -0.01 & 0.00 \\
\hline RC1 (8) & 30 & 5 & 36474.96 & 1132.50 & 36715.80 & $10800.00^{*}$ & 1132.50 & 36717.50 & 129.89 & 12.69 & 0.00 & 0.00 \\
\hline RC2 (8) & 30 & 5 & 36199.06 & 1131.25 & 36555.58 & $10800.00^{*}$ & 1131.25 & 36572.23 & 132.83 & 13.18 & 0.05 & 0.00 \\
\hline Avg & & & & & & 9359.53 & & & 133.55 & 13.05 & -0.01 & 0.00 \\
\hline
\end{tabular}

Table 7: Average results on medium- and large-size instances

\begin{tabular}{|c|c|c|c|c|c|c|c|c|c|c|c|c|}
\hline Instance set & $\left|\mathcal{N}_{c}\right|$ & $\left|\mathcal{N}_{0}\right|$ & $E_{4}^{v}$ & & & & HESA & & & & & \\
\hline & & & LP relaxation & Vehicle cost & Total cost & Time (s.) & Vehicle cost & Total cost & Total time (s.) & Time (s.) & $\operatorname{Dev}_{T C}$ & $\operatorname{Dev}_{V C}$ \\
\hline C1 (9) & 50 & 7 & 41386.56 & 2722.22 & 41875.93 & 9644.33 & 2722.22 & 41910.79 & 934.92 & 93.09 & 0.08 & 0.00 \\
\hline C2 (8) & 50 & 7 & 92446.10 & 2975.00 & 93394.71 & 9450.78 & 2400.00 & 92822.45 & 996.13 & 99.21 & -0.61 & -18.68 \\
\hline R1 (12) & 50 & 7 & 38870.22 & 1350.00 & 39722.87 & $10800.00^{*}$ & 1301.67 & 39488.63 & 937.20 & 93.32 & -0.58 & -3.42 \\
\hline R2 (11) & 50 & 7 & 175518.42 & 1540.91 & 176329.57 & $10800.00^{*}$ & 1350.00 & 175991.78 & 931.02 & 92.70 & -0.19 & -9.90 \\
\hline RC1 (8) & 50 & 7 & 19574.34 & 2081.25 & 22807.06 & $10800.00^{*}$ & 1938.75 & 20485.74 & 769.46 & 76.55 & -6.42 & -6.61 \\
\hline RC2 (8) & 50 & 7 & 87065.42 & 2181.25 & 88183.79 & $10800.00^{*}$ & 1868.75 & 87536.04 & 860.16 & 85.62 & -0.73 & -9.87 \\
\hline Avg & & & & & & 10382.52 & & & 904.82 & 90.08 & -1.41 & -8.08 \\
\hline C1 (9) & 75 & 8 & 82857.15 & 4511.11 & 83662.53 & $10800.00^{*}$ & 4288.89 & 83479.50 & 1739.49 & 173.35 & -0.22 & -4.20 \\
\hline C2 (8) & 75 & 8 & 188792.53 & 7075.00 & 192980.52 & $10800.00^{*}$ & 3800.00 & 189374.75 & 1662.91 & 165.39 & -1.84 & -37.46 \\
\hline R1 (12) & 75 & 8 & 38056.29 & 4041.67 & 64165.06 & $10800.00^{*}$ & 1969.17 & 39939.26 & 1726.14 & 172.11 & -23.09 & -41.79 \\
\hline R2 (11) & 75 & 8 & 174160.82 & 5245.45 & 178597.02 & $10800.00^{*}$ & 1800.00 & 174601.27 & 1621.98 & 161.90 & -2.23 & -60.60 \\
\hline RC1 (8) & 75 & 8 & 38233.86 & 3086.25 & 39523.52 & $10800.00^{*}$ & 2595.00 & 38768.89 & 1741.39 & 174.04 & -1.90 & -14.64 \\
\hline RC2 (8) & 75 & 8 & 173677.24 & 4175.00 & 176872.38 & $10800.00^{*}$ & 2450.00 & 174367.88 & 1608.39 & 160.14 & -1.41 & -33.89 \\
\hline Avg & & & & & & $10800.00^{*}$ & & & 1683.38 & 167.82 & -5.11 & -32.10 \\
\hline C1 (9) & 100 & 10 & 84345.45 & 7044.44 & 86863.29 & $10800.00^{*}$ & 5788.89 & 85234.48 & 3082.24 & 307.82 & -1.83 & -13.61 \\
\hline C2 (8) & 100 & 10 & 190077.54 & 22887.50 & 330436.31 & $10800.00^{*}$ & 5200.00 & 190862.38 & 3083.79 & 308.28 & -17.58 & -56.77 \\
\hline R1 (12) & 100 & 10 & 39151.72 & 9831.67 & 166296.26 & $10800.00^{*}$ & 2648.33 & 40972.43 & 2902.51 & 289.95 & -65.67 & -66.69 \\
\hline R2 (11) & 100 & 10 & 174794.11 & 12068.18 & 185688.94 & $10800.00^{*}$ & 2250.00 & 175171.82 & 2934.80 & 292.88 & -5.64 & -80.01 \\
\hline RC1 (8) & 100 & 10 & 39186.42 & 6123.75 & 49304.23 & $10800.00^{*}$ & 3405.00 & 39889.18 & 3062.54 & 306.05 & -15.53 & -40.49 \\
\hline RC2 (8) & 100 & 10 & 174430.65 & 12568.75 & 268749.92 & $10800.00^{*}$ & 2962.50 & 175057.75 & 2956.81 & 294.88 & -13.35 & -65.35 \\
\hline Avg & & & & & & $10800.00^{*}$ & & & 3003.78 & 299.98 & -19.93 & -53.82 \\
\hline
\end{tabular}




\section{Conclusions}

This paper has introduced the FSMLRPTW, a complex integrated logistics problem which, to our knowledge, was studied here for the first time, and has described a hybrid evolutionary search algorithm tailored to the problem. We have introduced several algorithmic procedures specific to the FSMLRPTW, namely, the location-heterogeneous adaptive large neighborhood search procedure equipped with a range of new operators as the main EDUCATION procedure within the search. We have also developed an INITIALIZATION procedure to create initial solutions, a PARTITION procedure for offspring solutions, and a new diversification scheme through the MUTATION procedure of solutions. Computational results on a new set of benchmark instances of up to 100 nodes and 10 potential depots were presented, which indicate that the proposed algorithm is able to identify solutions within $0.05 \%$ of optimality for small size instances and yields better solutions for larger instances as compared to an off-the-shelf solver. The running times of the algorithm are so that it can be used in practical applications.

\section{Acknowledgements}

The authors gratefully acknowledge funding provided by the Southampton Business School of University of Southampton and by the Canadian Natural Sciences and Engineering Research Council under grants 39682-10 and 436014-2013. Thanks are due to two referees for their valuable comments.

\section{Appendix}

Table A.1 presents the characteristics of the FSMLRPTW instances. Tables A.2-A.9 present the detailed results on all benchmark instances for the FSMLRPTW instances. 
Table A.1: The FSMLRPTW benchmark instances

\begin{tabular}{|c|c|c|c|c|c|c|c|c|c|c|c|c|}
\hline Data set & Depot & Cost & $\mathrm{Cap}_{10}$ & $\operatorname{Cap}_{15}$ & $\mathrm{Cap}_{20}$ & $\mathrm{Cap}_{25}$ & $\mathrm{Cap}_{30}$ & $\mathrm{Cap}_{50}$ & $\mathrm{Cap}_{75}$ & $\mathrm{Cap}_{100}$ & $X$ & $\mathrm{Y}$ \\
\hline \multirow[t]{10}{*}{$\mathrm{C} 1$} & 1 & 40000 & 100 & 200 & 210 & 320 & 400 & 610 & 850 & 990 & 40 & 50 \\
\hline & 2 & 45000 & 90 & 170 & 205 & 280 & 410 & 580 & 810 & 800 & 64 & 13 \\
\hline & 3 & 42000 & 110 & 210 & 220 & 300 & 370 & 620 & 820 & 900 & 35 & 79 \\
\hline & 4 & 41000 & - & 150 & 190 & 220 & 360 & 540 & 860 & 850 & 44 & 57 \\
\hline & 5 & 48000 & - & - & 215 & 290 & 390 & 550 & 880 & 840 & 29 & 40 \\
\hline & 6 & 50000 & - & - & - & - & - & 600 & 900 & 970 & 18 & 82 \\
\hline & 7 & 38000 & - & - & - & - & - & 590 & 830 & 1000 & 63 & 93 \\
\hline & 8 & 49000 & - & - & - & - & - & - & 870 & 910 & 85 & 8 \\
\hline & 9 & 47000 & - & - & - & - & - & - & - & 930 & 11 & 63 \\
\hline & 10 & 46000 & - & - & - & - & - & - & - & 780 & 37 & 17 \\
\hline \multirow[t]{10}{*}{$\mathrm{C} 2$} & 1 & 90000 & 110 & 220 & 240 & 340 & 430 & 640 & 880 & 950 & 40 & 50 \\
\hline & 2 & 100000 & 100 & 180 & 230 & 290 & 410 & 610 & 840 & 800 & 8 & 95 \\
\hline & 3 & 120000 & 120 & 210 & 240 & 310 & 380 & 630 & 830 & 1010 & 91 & 46 \\
\hline & 4 & 95000 & - & 160 & 210 & 230 & 360 & 660 & 880 & 970 & 35 & 43 \\
\hline & 5 & 105000 & - & - & 230 & 320 & 420 & 580 & 900 & 920 & 20 & 69 \\
\hline & 6 & 97000 & - & - & - & - & - & 620 & 930 & 990 & 51 & 100 \\
\hline & 7 & 115000 & - & - & - & - & - & 640 & 870 & 1030 & 29 & 28 \\
\hline & 8 & 112000 & - & - & - & - & - & - & 890 & 930 & 60 & 43 \\
\hline & 9 & 99000 & - & - & - & - & - & - & - & 870 & 98 & 97 \\
\hline & 10 & 117000 & - & - & - & - & - & - & - & 890 & 96 & 42 \\
\hline \multirow[t]{10}{*}{ R1 } & 1 & 20000 & 80 & 150 & 160 & 300 & 330 & 490 & 750 & 960 & 35 & 35 \\
\hline & 2 & 19000 & 95 & 110 & 140 & 280 & 280 & 440 & 690 & 750 & 10 & 54 \\
\hline & 3 & 22000 & 85 & 130 & 140 & 270 & 300 & 420 & 610 & 910 & 52 & 56 \\
\hline & 4 & 21000 & - & 160 & 150 & 290 & 310 & 510 & 650 & 820 & 46 & 60 \\
\hline & 5 & 18000 & - & - & 170 & 310 & 270 & 500 & 760 & 720 & 81 & 24 \\
\hline & 6 & 23000 & - & - & - & - & - & 390 & 740 & 790 & 11 & 59 \\
\hline & 7 & 24000 & - & - & - & - & - & 460 & 590 & 1000 & 94 & 40 \\
\hline & 8 & 17000 & - & - & - & - & - & - & 550 & 800 & 78 & 77 \\
\hline & 9 & 25000 & - & - & - & - & - & - & - & 790 & 88 & 66 \\
\hline & 10 & 20000 & - & - & - & - & - & - & - & 890 & 72 & 49 \\
\hline \multirow[t]{10}{*}{$\mathrm{R} 2$} & 1 & 85000 & 100 & 170 & 190 & 305 & 340 & 530 & 780 & 1020 & 35 & 35 \\
\hline & 2 & 90000 & 105 & 120 & 160 & 290 & 300 & 480 & 730 & 810 & 92 & 77 \\
\hline & 3 & 94000 & 90 & 140 & 150 & 300 & 310 & 470 & 650 & 720 & 82 & 17 \\
\hline & 4 & 89000 & - & 180 & 160 & 315 & 330 & 530 & 670 & 790 & 25 & 82 \\
\hline & 5 & 100000 & - & - & 190 & 310 & 280 & 520 & 790 & 890 & 64 & 17 \\
\hline & 6 & 92000 & - & - & - & - & - & 450 & 750 & 1070 & 100 & 87 \\
\hline & 7 & 97000 & - & - & - & - & - & 480 & 640 & 740 & 10 & 72 \\
\hline & 8 & 87000 & - & - & - & - & - & - & 630 & 700 & 3 & 51 \\
\hline & 9 & 99000 & - & - & - & - & - & - & - & 1100 & 8 & 99 \\
\hline & 10 & 96000 & - & - & - & - & - & - & - & 760 & 64 & 60 \\
\hline \multirow[t]{10}{*}{ RC1 } & 1 & 18000 & 160 & 240 & 250 & 310 & 420 & 700 & 860 & 1050 & 40 & 50 \\
\hline & 2 & 19000 & 180 & 250 & 260 & 270 & 370 & 680 & 790 & 900 & 15 & 52 \\
\hline & 3 & 17000 & 150 & 230 & 220 & 320 & 390 & 710 & 780 & 1090 & 40 & 3 \\
\hline & 4 & 21000 & - & 210 & 240 & 290 & 430 & 640 & 870 & 850 & 24 & 93 \\
\hline & 5 & 26000 & - & - & 230 & 340 & 400 & 630 & 840 & 790 & 50 & 76 \\
\hline & 6 & 24000 & - & - & - & - & - & 640 & 730 & 940 & 62 & 60 \\
\hline & 7 & 23000 & - & - & - & - & - & 670 & 800 & 970 & 79 & 100 \\
\hline & 8 & 19000 & - & - & - & - & - & - & 850 & 1180 & 10 & 95 \\
\hline & 9 & 20000 & - & - & - & - & - & - & - & 900 & 80 & 11 \\
\hline & 10 & 25000 & - & - & - & - & - & - & - & 1020 & 87 & 75 \\
\hline \multirow[t]{10}{*}{ RC2 } & 1 & 86000 & 180 & 270 & 320 & 420 & 420 & 720 & 900 & 1300 & 40 & 50 \\
\hline & 2 & 91000 & 190 & 260 & 300 & 360 & 370 & 710 & 830 & 1200 & 86 & 37 \\
\hline & 3 & 87000 & 160 & 240 & 270 & 380 & 390 & 740 & 800 & 900 & 23 & 94 \\
\hline & 4 & 99000 & - & 230 & 290 & 370 & 430 & 690 & 890 & 800 & 55 & 100 \\
\hline & 5 & 96000 & - & - & 310 & 410 & 400 & 680 & 860 & 1080 & 28 & 92 \\
\hline & 6 & 100000 & - & - & - & - & - & 670 & 760 & 780 & 68 & 52 \\
\hline & 7 & 85000 & - & - & - & - & - & 710 & 830 & 1090 & 72 & 19 \\
\hline & 8 & 94000 & - & - & - & - & - & - & 870 & 1240 & 34 & 61 \\
\hline & 9 & 93000 & - & - & - & - & - & - & - & 900 & 26 & 88 \\
\hline & 10 & 97000 & - & - & - & - & - & - & - & 1100 & 61 & 44 \\
\hline
\end{tabular}


Table A.2: Results on the 10-customer instances

\begin{tabular}{|c|c|c|c|c|c|c|c|c|c|c|c|}
\hline \multirow[t]{2}{*}{ Instance set } & \multirow[t]{2}{*}{$\left|\mathcal{N}_{c}\right|$} & \multirow[t]{2}{*}{$\left|\mathcal{N}_{0}\right|$} & \multicolumn{4}{|l|}{$E_{4}^{v}$} & \multicolumn{5}{|l|}{ HESA } \\
\hline & & & LP relaxation & Vehicle cost & Total cost & Time (s.) & Vehicle cost & Total cost & Time (s.) & $\operatorname{Dev}_{T C}$ & $\operatorname{Dev}_{V C}$ \\
\hline C101 & 10 & 3 & 82504.85 & 600.00 & 82675.76 & 0.04 & 600.00 & 82675.76 & 4.59 & 0.00 & 0.00 \\
\hline C102 & 10 & 3 & 82504.85 & 600.00 & 82673.74 & 0.68 & 600.00 & 82673.74 & 4.91 & 0.00 & 0.00 \\
\hline C103 & 10 & 3 & 82504.85 & 600.00 & 82673.74 & 0.68 & 600.00 & 82673.74 & 2.83 & 0.00 & 0.00 \\
\hline C104 & 10 & 3 & 82504.85 & 600.00 & 82673.74 & 1.46 & 600.00 & 82673.74 & 2.60 & 0.00 & 0.00 \\
\hline C105 & 10 & 3 & 82504.85 & 600.00 & 82675.76 & 0.04 & 600.00 & 82675.76 & 3.82 & 0.00 & 0.00 \\
\hline C106 & 10 & 3 & 82504.85 & 600.00 & 82675.76 & 0.03 & 600.00 & 82675.76 & 3.96 & 0.00 & 0.00 \\
\hline C107 & 10 & 3 & 82504.85 & 600.00 & 82675.76 & 0.03 & 600.00 & 82675.76 & 4.32 & 0.00 & 0.00 \\
\hline C108 & 10 & 3 & 82504.85 & 600.00 & 82673.74 & 0.17 & 600.00 & 82673.74 & 2.03 & 0.00 & 0.00 \\
\hline C109 & 10 & 3 & 82504.85 & 600.00 & 82673.74 & 0.25 & 600.00 & 82673.74 & 3.06 & 0.00 & 0.00 \\
\hline C201 & 10 & 3 & 191118.19 & 2000.00 & 192212.53 & 0.06 & 2000.00 & 192212.53 & 4.87 & 0.00 & 0.00 \\
\hline C202 & 10 & 3 & 191118.19 & 2000.00 & 192205.36 & 5.04 & 2000.00 & 192205.36 & 1.57 & 0.00 & 0.00 \\
\hline C203 & 10 & 3 & 191118.19 & 2000.00 & 192205.36 & 5.03 & 2000.00 & 192205.36 & 1.94 & 0.00 & 0.00 \\
\hline C204 & 10 & 3 & 191118.19 & 2000.00 & 192195.93 & 4.97 & 2000.00 & 192195.93 & 3.57 & 0.00 & 0.00 \\
\hline C205 & 10 & 3 & 191118.19 & 2000.00 & 192207.74 & 0.19 & 2000.00 & 192207.74 & 4.51 & 0.00 & 0.00 \\
\hline C206 & 10 & 3 & 191118.19 & 2000.00 & 192195.93 & 0.07 & 2000.00 & 192195.93 & 3.22 & 0.00 & 0.00 \\
\hline C207 & 10 & 3 & 191118.19 & 2000.00 & 192195.93 & 0.16 & 2000.00 & 192195.93 & 3.13 & 0.00 & 0.00 \\
\hline C208 & 10 & 3 & 191118.19 & 2000.00 & 192195.93 & 1.00 & 2000.00 & 192195.93 & 3.69 & 0.00 & 0.00 \\
\hline R101 & 10 & 3 & 39382.04 & 260.00 & 39523.07 & 0.11 & 260.00 & 39523.07 & 1.18 & 0.00 & 0.00 \\
\hline R102 & 10 & 3 & 39382.04 & 210.00 & 39459.59 & 100.29 & 210.00 & 39459.59 & 4.53 & 0.00 & 0.00 \\
\hline R103 & 10 & 3 & 39382.04 & 210.00 & 39459.59 & 100.31 & 210.00 & 39459.59 & 3.80 & 0.00 & 0.00 \\
\hline R104 & 10 & 3 & 39382.04 & 220.00 & 39446.25 & 195.61 & 220.00 & 39446.25 & 4.18 & 0.00 & 0.00 \\
\hline R105 & 10 & 3 & 39382.04 & 210.00 & 39498.13 & 0.20 & 210.00 & 39498.13 & 1.99 & 0.00 & 0.00 \\
\hline R106 & 10 & 3 & 39382.04 & 210.00 & 39459.59 & 204.49 & 210.00 & 39459.59 & 4.18 & 0.00 & 0.00 \\
\hline R107 & 10 & 3 & 39382.04 & 210.00 & 39459.59 & 204.84 & 210.00 & 39459.59 & 1.99 & 0.00 & 0.00 \\
\hline R108 & 10 & 3 & 39382.04 & 220.00 & 39446.25 & 202.89 & 220.00 & 39446.25 & 2.33 & 0.00 & 0.00 \\
\hline R109 & 10 & 3 & 39382.04 & 210.00 & 39483.11 & 3.78 & 210.00 & 39483.11 & 1.97 & 0.00 & 0.00 \\
\hline R110 & 10 & 3 & 39382.04 & 210.00 & 39452.56 & 33.73 & 210.00 & 39452.56 & 4.48 & 0.00 & 0.00 \\
\hline R111 & 10 & 3 & 39382.04 & 210.00 & 39462.11 & 205.15 & 210.00 & 39462.11 & 1.40 & 0.00 & 0.00 \\
\hline R112 & 10 & 3 & 39382.04 & 220.00 & 39446.25 & 292.26 & 220.00 & 39446.25 & 4.11 & 0.00 & 0.00 \\
\hline R201 & 10 & 3 & 175618.55 & 900.00 & 176202.33 & 0.17 & 900.00 & 176202.33 & 4.04 & 0.00 & 0.00 \\
\hline R202 & 10 & 3 & 175618.55 & 900.00 & 176152.08 & 0.76 & 900.00 & 176152.08 & 2.65 & 0.00 & 0.00 \\
\hline R203 & 10 & 3 & 175618.55 & 900.00 & 176152.08 & 0.76 & 900.00 & 176152.08 & 3.54 & 0.00 & 0.00 \\
\hline R204 & 10 & 3 & 175618.55 & 900.00 & 176147.33 & 1.40 & 900.00 & 176147.33 & 4.91 & 0.00 & 0.00 \\
\hline R205 & 10 & 3 & 175618.55 & 900.00 & 176175.45 & 0.67 & 900.00 & 176175.45 & 3.17 & 0.00 & 0.00 \\
\hline R206 & 10 & 3 & 175618.55 & 900.00 & 176147.33 & 1.45 & 900.00 & 176147.33 & 2.20 & 0.00 & 0.00 \\
\hline R207 & 10 & 3 & 175618.55 & 900.00 & 176147.33 & 1.44 & 900.00 & 176147.33 & 1.12 & 0.00 & 0.00 \\
\hline R208 & 10 & 3 & 175618.55 & 900.00 & 176147.33 & 0.95 & 900.00 & 176147.33 & 2.77 & 0.00 & 0.00 \\
\hline R209 & 10 & 3 & 175618.55 & 900.00 & 176162.07 & 0.41 & 900.00 & 176162.07 & 3.81 & 0.00 & 0.00 \\
\hline R210 & 10 & 3 & 175618.55 & 900.00 & 176152.08 & 1.84 & 900.00 & 176152.08 & 4.99 & 0.00 & 0.00 \\
\hline R211 & 10 & 3 & 175618.55 & 900.00 & 176152.08 & 0.75 & 900.00 & 176152.08 & 1.79 & 0.00 & 0.00 \\
\hline RC101 & 10 & 3 & 35614.85 & 450.00 & 35766.20 & 1.47 & 450.00 & 35766.20 & 4.55 & 0.00 & 0.00 \\
\hline RC102 & 10 & 3 & 35614.85 & 450.00 & 35704.15 & 12.61 & 450.00 & 35704.15 & 1.98 & 0.00 & 0.00 \\
\hline RC103 & 10 & 3 & 35614.85 & 450.00 & 35704.15 & 12.60 & 450.00 & 35704.15 & 3.71 & 0.00 & 0.00 \\
\hline RC104 & 10 & 3 & 35614.85 & 450.00 & 35704.15 & 6.50 & 450.00 & 35704.15 & 1.83 & 0.00 & 0.00 \\
\hline RC105 & 10 & 3 & 35614.85 & 450.00 & 35731.49 & 36.21 & 450.00 & 35731.49 & 2.28 & 0.00 & 0.00 \\
\hline RC106 & 10 & 3 & 35614.85 & 450.00 & 35716.72 & 6.38 & 450.00 & 35716.72 & 4.94 & 0.00 & 0.00 \\
\hline RC107 & 10 & 3 & 35614.85 & 450.00 & 35704.15 & 3.39 & 450.00 & 35704.15 & 4.12 & 0.00 & 0.00 \\
\hline RC108 & 10 & 3 & 35614.85 & 450.00 & 35704.15 & 7.02 & 450.00 & 35704.15 & 2.08 & 0.00 & 0.00 \\
\hline RC201 & 10 & 3 & 173434.98 & 450.00 & 173626.44 & 2.62 & 450.00 & 173626.44 & 2.28 & 0.00 & 0.00 \\
\hline RC202 & 10 & 3 & 173434.98 & 450.00 & 173622.36 & 8.67 & 450.00 & 173622.36 & 2.04 & 0.00 & 0.00 \\
\hline RC203 & 10 & 3 & 173434.98 & 450.00 & 173622.36 & 8.67 & 450.00 & 173622.36 & 4.61 & 0.00 & 0.00 \\
\hline RC204 & 10 & 3 & 173434.98 & 450.00 & 173620.76 & 15.82 & 450.00 & 173620.76 & 1.62 & 0.00 & 0.00 \\
\hline RC205 & 10 & 3 & 173434.98 & 450.00 & 173623.73 & 8.32 & 450.00 & 173623.73 & 1.89 & 0.00 & 0.00 \\
\hline RC206 & 10 & 3 & 173434.98 & 450.00 & 173620.76 & 5.30 & 450.00 & 173620.76 & 3.34 & 0.00 & 0.00 \\
\hline RC207 & 10 & 3 & 173434.98 & 450.00 & 173620.76 & 56.11 & 450.00 & 173620.76 & 3.72 & 0.00 & 0.00 \\
\hline RC208 & 10 & 3 & 173434.98 & 450.00 & 173620.76 & 12.57 & 450.00 & 173620.76 & 3.07 & 0.00 & 0.00 \\
\hline
\end{tabular}


Table A.3: Results on the 15-customer instances

\begin{tabular}{|c|c|c|c|c|c|c|c|c|c|c|c|}
\hline \multirow[t]{2}{*}{ Instance set } & \multirow[t]{2}{*}{$\left|\mathcal{N}_{c}\right|$} & \multirow[t]{2}{*}{$\left|\mathcal{N}_{0}\right|$} & \multicolumn{4}{|l|}{$E_{4}^{v}$} & \multicolumn{5}{|l|}{ HESA } \\
\hline & & & LP relaxation & Vehicle cost & Total cost & Time (s.) & Vehicle cost & Total cost & Time (s.) & $\operatorname{Dev}_{T C}$ & $\operatorname{Dev}_{V C}$ \\
\hline C101 & 15 & 4 & 81889.59 & 900.00 & 82060.38 & 0.36 & 900.00 & 82060.38 & 5.56 & 0.00 & 0.00 \\
\hline C102 & 15 & 4 & 81889.59 & 900.00 & 82059.67 & 5.10 & 900.00 & 82059.67 & 4.09 & 0.00 & 0.00 \\
\hline C103 & 15 & 4 & 81889.59 & 900.00 & 82059.67 & 14.04 & 900.00 & 82059.67 & 5.66 & 0.00 & 0.00 \\
\hline C104 & 15 & 4 & 81889.59 & 900.00 & 82058.48 & 14.50 & 900.00 & 82058.48 & 3.34 & 0.00 & 0.00 \\
\hline C105 & 15 & 4 & 81889.59 & 900.00 & 82060.38 & 0.66 & 900.00 & 82060.38 & 5.35 & 0.00 & 0.00 \\
\hline C106 & 15 & 4 & 81889.59 & 900.00 & 82060.38 & 0.25 & 900.00 & 82060.38 & 3.76 & 0.00 & 0.00 \\
\hline C107 & 15 & 4 & 81889.59 & 900.00 & 82060.38 & 0.63 & 900.00 & 82060.38 & 2.15 & 0.00 & 0.00 \\
\hline C108 & 15 & 4 & 81889.59 & 900.00 & 82060.38 & 3.71 & 900.00 & 82060.38 & 5.98 & 0.00 & 0.00 \\
\hline C109 & 15 & 4 & 81889.59 & 900.00 & 82059.54 & 10.38 & 900.00 & 82059.54 & 4.73 & 0.00 & 0.00 \\
\hline C201 & 15 & 4 & 186126.81 & 2000.00 & 187199.20 & 0.24 & 2000.00 & 187199.20 & 2.64 & 0.00 & 0.00 \\
\hline C202 & 15 & 4 & 186126.81 & 2000.00 & 187190.12 & 11.17 & 2000.00 & 187190.12 & 4.56 & 0.00 & 0.00 \\
\hline C203 & 15 & 4 & 186126.81 & 2000.00 & 187190.12 & 12.93 & 2000.00 & 187190.12 & 4.26 & 0.00 & 0.00 \\
\hline C204 & 15 & 4 & 186126.81 & 2000.00 & 187190.12 & 26.52 & 2000.00 & 187190.12 & 3.58 & 0.00 & 0.00 \\
\hline C205 & 15 & 4 & 186126.81 & 2000.00 & 187194.86 & 2.38 & 2000.00 & 187194.86 & 3.31 & 0.00 & 0.00 \\
\hline C206 & 15 & 4 & 186126.81 & 2000.00 & 187194.86 & 3.17 & 2000.00 & 187194.86 & 2.84 & 0.00 & 0.00 \\
\hline C207 & 15 & 4 & 186126.81 & 2000.00 & 187194.86 & 9.43 & 2000.00 & 187194.86 & 5.18 & 0.00 & 0.00 \\
\hline C208 & 15 & 4 & 186126.81 & 2000.00 & 187194.86 & 10.29 & 2000.00 & 187194.86 & 3.37 & 0.00 & 0.00 \\
\hline R101 & 15 & 4 & 39590.64 & 340.00 & 39753.66 & 0.32 & 340.00 & 39753.66 & 3.61 & 0.00 & 0.00 \\
\hline R102 & 15 & 4 & 39590.64 & 400.00 & 39714.93 & 3465.17 & 400.00 & 39714.93 & 2.24 & 0.00 & 0.00 \\
\hline R103 & 15 & 4 & 39590.64 & 400.00 & 39714.93 & 9902.70 & 400.00 & 39714.93 & 2.17 & 0.00 & 0.00 \\
\hline R104 & 15 & 4 & 39590.64 & 340.00 & 39691.17 & $10800.00^{*}$ & 340.00 & 39692.00 & 4.28 & 0.00 & 0.00 \\
\hline R105 & 15 & 4 & 39590.64 & 370.00 & 39748.26 & 14.69 & 370.00 & 39748.26 & 3.56 & 0.00 & 0.00 \\
\hline R106 & 15 & 4 & 39590.64 & 350.00 & 39692.47 & 3703.37 & 350.00 & 39692.47 & 4.92 & 0.00 & 0.00 \\
\hline R107 & 15 & 4 & 39590.64 & 350.00 & 39692.47 & 8024.12 & 350.00 & 39692.47 & 2.40 & 0.00 & 0.00 \\
\hline R108 & 15 & 4 & 39590.64 & 350.00 & 39678.30 & $10800.00^{*}$ & 350.00 & 39678.30 & 4.91 & 0.00 & 0.00 \\
\hline R109 & 15 & 4 & 39590.64 & 350.00 & 39699.94 & 239.23 & 350.00 & 39699.94 & 4.38 & 0.00 & 0.00 \\
\hline R110 & 15 & 4 & 39590.64 & 360.00 & 39683.72 & $10800.00^{*}$ & 360.00 & 39683.72 & 5.37 & 0.00 & 0.00 \\
\hline R111 & 15 & 4 & 39590.64 & 350.00 & 39692.47 & 9580.68 & 350.00 & 39692.47 & 2.49 & 0.00 & 0.00 \\
\hline R112 & 15 & 4 & 39590.64 & 360.00 & 39665.72 & 8609.80 & 360.00 & 39665.72 & 5.95 & 0.00 & 0.00 \\
\hline R201 & 15 & 4 & 174662.67 & 900.00 & 175234.54 & 0.30 & 900.00 & 175234.54 & 4.97 & 0.00 & 0.00 \\
\hline R202 & 15 & 4 & 174662.67 & 900.00 & 175188.92 & 131.56 & 900.00 & 175188.92 & 4.12 & 0.00 & 0.00 \\
\hline R203 & 15 & 4 & 174662.67 & 900.00 & 175188.92 & 82.87 & 900.00 & 175188.92 & 3.80 & 0.00 & 0.00 \\
\hline R204 & 15 & 4 & 174662.67 & 900.00 & 175188.16 & 300.89 & 900.00 & 175188.16 & 2.03 & 0.00 & 0.00 \\
\hline R205 & 15 & 4 & 174662.67 & 900.00 & 175198.70 & 0.48 & 900.00 & 175198.70 & 2.94 & 0.00 & 0.00 \\
\hline R206 & 15 & 4 & 174662.67 & 900.00 & 175188.16 & 60.86 & 900.00 & 175188.16 & 2.43 & 0.00 & 0.00 \\
\hline R207 & 15 & 4 & 174662.67 & 900.00 & 175188.16 & 125.80 & 900.00 & 175188.16 & 4.40 & 0.00 & 0.00 \\
\hline R208 & 15 & 4 & 174662.67 & 900.00 & 175186.29 & 146.41 & 900.00 & 175186.29 & 5.54 & 0.00 & 0.00 \\
\hline R209 & 15 & 4 & 174662.67 & 900.00 & 175185.29 & 88.86 & 900.00 & 175185.29 & 3.01 & 0.00 & 0.00 \\
\hline R210 & 15 & 4 & 174662.67 & 900.00 & 175188.92 & 138.54 & 900.00 & 175188.92 & 5.39 & 0.00 & 0.00 \\
\hline R211 & 15 & 4 & 174662.67 & 900.00 & 175155.24 & 9.32 & 900.00 & 175155.24 & 4.08 & 0.00 & 0.00 \\
\hline RC101 & 15 & 4 & 35843.17 & 660.00 & 36000.10 & 68.82 & 660.00 & 36000.10 & 3.28 & 0.00 & 0.00 \\
\hline RC102 & 15 & 4 & 35843.17 & 660.00 & 35959.01 & 403.37 & 660.00 & 35959.01 & 2.44 & 0.00 & 0.00 \\
\hline RC103 & 15 & 4 & 35843.17 & 660.00 & 35954.04 & 1735.09 & 660.00 & 35954.04 & 4.44 & 0.00 & 0.00 \\
\hline RC104 & 15 & 4 & 35843.17 & 660.00 & 35951.15 & 7053.61 & 660.00 & 35951.15 & 2.61 & 0.00 & 0.00 \\
\hline RC105 & 15 & 4 & 35843.17 & 660.00 & 36003.22 & 6482.74 & 660.00 & 36003.22 & 3.35 & 0.00 & 0.00 \\
\hline RC106 & 15 & 4 & 35843.17 & 660.00 & 35950.90 & 842.69 & 660.00 & 35950.90 & 4.96 & 0.00 & 0.00 \\
\hline RC107 & 15 & 4 & 35843.17 & 660.00 & 35936.06 & 8415.18 & 660.00 & 35936.06 & 5.27 & 0.00 & 0.00 \\
\hline RC108 & 15 & 4 & 35843.17 & 660.00 & 35933.94 & 2751.09 & 660.00 & 35933.94 & 2.45 & 0.00 & 0.00 \\
\hline RC201 & 15 & 4 & 173626.06 & 650.00 & 173836.24 & 372.04 & 650.00 & 173836.24 & 3.63 & 0.00 & 0.00 \\
\hline RC202 & 15 & 4 & 173626.06 & 650.00 & 173829.47 & $10800.00^{*}$ & 650.00 & 173829.47 & 5.14 & 0.00 & 0.00 \\
\hline RC203 & 15 & 4 & 173626.06 & 650.00 & 173829.47 & $10800.00^{*}$ & 650.00 & 173829.47 & 2.48 & 0.00 & 0.00 \\
\hline RC204 & 15 & 4 & 173626.06 & 650.00 & 173823.01 & $10800.00^{*}$ & 650.00 & 173823.01 & 2.17 & 0.00 & 0.00 \\
\hline RC205 & 15 & 4 & 173626.06 & 650.00 & 173829.47 & $10800.00^{*}$ & 650.00 & 173829.47 & 5.64 & 0.00 & 0.00 \\
\hline RC206 & 15 & 4 & 173626.06 & 650.00 & 173824.84 & $10800.00^{*}$ & 650.00 & 173824.84 & 4.36 & 0.00 & 0.00 \\
\hline RC207 & 15 & 4 & 173626.06 & 650.00 & 173819.37 & $10800.00^{*}$ & 650.00 & 173819.37 & 4.90 & 0.00 & 0.00 \\
\hline RC208 & 15 & 4 & 173626.06 & 650.00 & 173817.77 & $10800.00^{*}$ & 650.00 & 173817.77 & 4.91 & 0.00 & 0.00 \\
\hline
\end{tabular}


Table A.4: Results on the 20-customer instances

\begin{tabular}{|c|c|c|c|c|c|c|c|c|c|c|c|}
\hline \multirow[t]{2}{*}{ Instance set } & \multirow[t]{2}{*}{$\left|\mathcal{N}_{c}\right|$} & \multirow[t]{2}{*}{$\left|\mathcal{N}_{0}\right|$} & \multicolumn{4}{|l|}{$E_{4}^{v}$} & \multicolumn{5}{|l|}{ HESA } \\
\hline & & & LP relaxation & Vehicle cost & Total cost & Time (s.) & Vehicle cost & Total cost & Time (s.) & $\operatorname{Dev}_{T C}$ & $\operatorname{Dev}_{V C}$ \\
\hline C101 & 20 & 5 & 82236.26 & 1200.00 & 82436.10 & 1.87 & 1200.00 & 82436.10 & 5.91 & 0.00 & 0.00 \\
\hline C102 & 20 & 5 & 82236.26 & 1200.00 & 82436.10 & 1382.38 & 1200.00 & 82436.10 & 8.04 & 0.00 & 0.00 \\
\hline C103 & 20 & 5 & 82236.26 & 1200.00 & 82436.10 & $10800.00^{*}$ & 1200.00 & 82436.10 & 4.30 & 0.00 & 0.00 \\
\hline C104 & 20 & 5 & 82236.26 & 1200.00 & 82435.04 & $10800.00^{*}$ & 1200.00 & 82566.30 & 7.41 & 0.16 & 0.00 \\
\hline C105 & 20 & 5 & 82236.26 & 1200.00 & 82436.10 & 2766.31 & 1200.00 & 82436.10 & 5.36 & 0.00 & 0.00 \\
\hline C106 & 20 & 5 & 82236.26 & 1200.00 & 82436.10 & 3.39 & 1200.00 & 82436.10 & 4.58 & 0.00 & 0.00 \\
\hline C107 & 20 & 5 & 82236.26 & 1200.00 & 82436.10 & 21.17 & 1200.00 & 82436.10 & 6.91 & 0.00 & 0.00 \\
\hline C108 & 20 & 5 & 82236.26 & 1200.00 & 82436.10 & $10800.00^{*}$ & 1200.00 & 82436.10 & 6.89 & 0.00 & 0.00 \\
\hline C109 & 20 & 5 & 82236.26 & 1200.00 & 82435.04 & $10800.00^{*}$ & 1200.00 & 82435.04 & 6.87 & 0.00 & 0.00 \\
\hline C201 & 20 & 5 & 186141.23 & 2000.00 & 187227.98 & 0.68 & 2000.00 & 187227.98 & 5.00 & 0.00 & 0.00 \\
\hline C202 & 20 & 5 & 186141.23 & 2000.00 & 187221.32 & 143.12 & 2000.00 & 187221.32 & 8.58 & 0.00 & 0.00 \\
\hline C203 & 20 & 5 & 186141.23 & 2000.00 & 187219.23 & 1655.04 & 2000.00 & 187219.23 & 5.03 & 0.00 & 0.00 \\
\hline C204 & 20 & 5 & 186141.23 & 2000.00 & 187218.28 & 2657.51 & 2000.00 & 187218.28 & 7.23 & 0.00 & 0.00 \\
\hline C205 & 20 & 5 & 186141.23 & 2000.00 & 187227.98 & 21.85 & 2000.00 & 187227.98 & 4.27 & 0.00 & 0.00 \\
\hline C206 & 20 & 5 & 186141.23 & 2000.00 & 187227.98 & 112.96 & 2000.00 & 187227.98 & 4.50 & 0.00 & 0.00 \\
\hline C207 & 20 & 5 & 186141.23 & 2000.00 & 187227.03 & 87.66 & 2000.00 & 187227.03 & 8.10 & 0.00 & 0.00 \\
\hline C208 & 20 & 5 & 186141.23 & 2000.00 & 187222.64 & 135.71 & 2000.00 & 187222.64 & 8.95 & 0.00 & 0.00 \\
\hline R101 & 20 & 5 & 37782.90 & 510.00 & 39130.26 & 3.58 & 510.00 & 39130.26 & 6.47 & 0.00 & 0.00 \\
\hline R102 & 20 & 5 & 37782.90 & 480.00 & 39055.61 & $10800.00^{*}$ & 480.00 & 39055.61 & 4.47 & 0.00 & 0.00 \\
\hline R103 & 20 & 5 & 37782.90 & 490.00 & 37973.97 & $10800.00^{*}$ & 490.00 & 37965.50 & 5.91 & -0.02 & 0.00 \\
\hline R104 & 20 & 5 & 37782.90 & 460.00 & 37931.84 & $10800.00^{*}$ & 460.00 & 37918.90 & 6.96 & -0.03 & 0.00 \\
\hline R105 & 20 & 5 & 37782.90 & 510.00 & 38054.09 & 221.14 & 510.00 & 38054.09 & 6.75 & 0.00 & 0.00 \\
\hline R106 & 20 & 5 & 37782.90 & 460.00 & 37965.26 & $10800.00^{*}$ & 460.00 & 37993.40 & 4.00 & 0.07 & 0.00 \\
\hline R107 & 20 & 5 & 37782.90 & 460.00 & 37940.97 & $10800.00^{*}$ & 460.00 & 37947.90 & 4.50 & 0.02 & 0.00 \\
\hline R108 & 20 & 5 & 37782.90 & 500.00 & 37927.36 & $10800.00^{*}$ & 500.00 & 37915.90 & 8.88 & -0.03 & 0.00 \\
\hline R109 & 20 & 5 & 37782.90 & 460.00 & 37931.63 & 1226.56 & 460.00 & 37931.63 & 7.41 & 0.00 & 0.00 \\
\hline R110 & 20 & 5 & 37782.90 & 460.00 & 37931.63 & $10800.00^{*}$ & 460.00 & 37931.63 & 8.51 & 0.00 & 0.00 \\
\hline R111 & 20 & 5 & 37782.90 & 460.00 & 37920.97 & $10800.00^{*}$ & 460.00 & 37920.97 & 4.44 & 0.00 & 0.00 \\
\hline R112 & 20 & 5 & 37782.90 & 500.00 & 37913.51 & $10800.00^{*}$ & 500.00 & 37906.70 & 5.87 & -0.02 & 0.00 \\
\hline R201 & 20 & 5 & 174699.42 & 900.00 & 175334.77 & 13.84 & 900.00 & 175335.00 & 6.83 & 0.00 & 0.00 \\
\hline R202 & 20 & 5 & 174699.40 & 900.00 & 175253.40 & 895.21 & 900.00 & 175253.40 & 6.29 & 0.00 & 0.00 \\
\hline R203 & 20 & 5 & 174699.40 & 900.00 & 175265.96 & $10800.00^{*}$ & 900.00 & 175253.00 & 6.12 & -0.01 & 0.00 \\
\hline R204 & 20 & 5 & 174699.40 & 900.00 & 175210.76 & 1169.83 & 900.00 & 175210.76 & 5.76 & 0.00 & 0.00 \\
\hline R205 & 20 & 5 & 174699.41 & 900.00 & 175242.43 & 2.47 & 900.00 & 175242.43 & 5.50 & 0.00 & 0.00 \\
\hline R206 & 20 & 5 & 174699.40 & 900.00 & 175225.04 & 170.27 & 900.00 & 175225.04 & 8.00 & 0.00 & 0.00 \\
\hline R207 & 20 & 5 & 174699.40 & 900.00 & 175225.04 & 3394.52 & 900.00 & 175225.04 & 5.81 & 0.00 & 0.00 \\
\hline R208 & 20 & 5 & 174699.40 & 900.00 & 175207.73 & 228.61 & 900.00 & 175207.73 & 6.40 & 0.00 & 0.00 \\
\hline R209 & 20 & 5 & 174699.41 & 900.00 & 175224.09 & 25.81 & 900.00 & 175224.09 & 4.39 & 0.00 & 0.00 \\
\hline R210 & 20 & 5 & 174699.40 & 900.00 & 175237.25 & 1028.92 & 900.00 & 175237.25 & 8.88 & 0.00 & 0.00 \\
\hline R211 & 20 & 5 & 174699.40 & 900.00 & 175208.53 & 782.13 & 900.00 & 175208.53 & 5.78 & 0.00 & 0.00 \\
\hline RC101 & 20 & 5 & 36075.96 & 870.00 & 36274.22 & 457.60 & 870.00 & 36288.50 & 5.30 & 0.04 & 0.00 \\
\hline RC102 & 20 & 5 & 36075.96 & 870.00 & 36241.99 & $10800.00^{*}$ & 870.00 & 36241.99 & 8.59 & 0.00 & 0.00 \\
\hline RC103 & 20 & 5 & 36075.96 & 870.00 & 36229.55 & $10800.00^{*}$ & 870.00 & 36236.60 & 7.17 & 0.02 & 0.00 \\
\hline RC104 & 20 & 5 & 36075.96 & 870.00 & 36229.32 & $10800.00^{*}$ & 870.00 & 36229.32 & 7.02 & 0.00 & 0.00 \\
\hline RC105 & 20 & 5 & 36075.96 & 870.00 & 36271.24 & $10800.00^{*}$ & 870.00 & 36281.50 & 6.87 & 0.03 & 0.00 \\
\hline RC106 & 20 & 5 & 36075.96 & 870.00 & 36248.47 & $10800.00^{*}$ & 870.00 & 36248.47 & 8.25 & 0.00 & 0.00 \\
\hline RC107 & 20 & 5 & 36075.96 & 870.00 & 36229.15 & $10800.00^{*}$ & 870.00 & 36229.15 & 7.59 & 0.00 & 0.00 \\
\hline RC108 & 20 & 5 & 36075.96 & 870.00 & 36215.68 & $10800.00^{*}$ & 870.00 & 36215.68 & 5.53 & 0.00 & 0.00 \\
\hline RC201 & 20 & 5 & 173864.43 & 800.00 & 174093.90 & $10800.00^{*}$ & 800.00 & 174093.90 & 6.05 & 0.00 & 0.00 \\
\hline RC202 & 20 & 5 & 173864.43 & 800.00 & 174086.74 & $10800.00^{*}$ & 800.00 & 174086.74 & 8.81 & 0.00 & 0.00 \\
\hline RC203 & 20 & 5 & 173864.43 & 800.00 & 174087.25 & $10800.00^{*}$ & 800.00 & 174081.00 & 8.14 & 0.00 & 0.00 \\
\hline RC204 & 20 & 5 & 173864.43 & 800.00 & 174069.80 & $10800.00^{*}$ & 800.00 & 174069.80 & 7.86 & 0.00 & 0.00 \\
\hline RC205 & 20 & 5 & 173864.43 & 800.00 & 174086.74 & $10800.00^{*}$ & 800.00 & 174086.74 & 6.56 & 0.00 & 0.00 \\
\hline RC206 & 20 & 5 & 173864.43 & 800.00 & 174082.07 & $10800.00^{*}$ & 800.00 & 174082.07 & 5.03 & 0.00 & 0.00 \\
\hline RC207 & 20 & 5 & 173864.43 & 800.00 & 174064.56 & $10800.00^{*}$ & 800.00 & 174064.56 & 7.90 & 0.00 & 0.00 \\
\hline RC208 & 20 & 5 & 173864.43 & 800.00 & 174064.56 & $10800.00^{*}$ & 800.00 & 174064.56 & 7.26 & 0.00 & 0.00 \\
\hline
\end{tabular}


Table A.5: Results on the 25-customer instances

\begin{tabular}{|c|c|c|c|c|c|c|c|c|c|c|c|}
\hline \multirow[t]{2}{*}{ Instance set } & \multirow[t]{2}{*}{$\left|\mathcal{N}_{c}\right|$} & \multirow[t]{2}{*}{$\left|\mathcal{N}_{0}\right|$} & \multicolumn{4}{|l|}{$E_{4}^{v}$} & \multicolumn{5}{|l|}{ HESA } \\
\hline & & & LP relaxation & Vehicle cost & Total cost & Time (s.) & Vehicle cost & Total cost & Time (s.) & $\operatorname{Dev}_{T C}$ & $\operatorname{Dev}_{V C}$ \\
\hline C101 & 25 & 5 & 82556.95 & 1500.00 & 82767.06 & 5.94 & 1500.00 & 82767.06 & 9.16 & 0.00 & 0.00 \\
\hline C102 & 25 & 5 & 82556.95 & 1500.00 & 82767.06 & 3941.41 & 1500.00 & 82767.06 & 10.76 & 0.00 & 0.00 \\
\hline C103 & 25 & 5 & 82556.95 & 1500.00 & 82766.82 & $10800.00^{*}$ & 1500.00 & 82773.80 & 9.91 & 0.01 & 0.00 \\
\hline C104 & 25 & 5 & 82556.95 & 1500.00 & 82764.87 & $10800.00^{*}$ & 1500.00 & 82902.10 & 10.35 & 0.17 & 0.00 \\
\hline C105 & 25 & 5 & 82556.95 & 1500.00 & 82767.06 & $10800.00^{*}$ & 1500.00 & 82767.06 & 9.75 & 0.00 & 0.00 \\
\hline C106 & 25 & 5 & 82556.95 & 1500.00 & 82767.06 & 6890.55 & 1500.00 & 82767.06 & 11.80 & 0.00 & 0.00 \\
\hline C107 & 25 & 5 & 82556.95 & 1500.00 & 82767.06 & $10800.00^{*}$ & 1500.00 & 82767.06 & 6.61 & 0.00 & 0.00 \\
\hline C108 & 25 & 5 & 82556.95 & 1500.00 & 82767.06 & $10800.00^{*}$ & 1500.00 & 82767.06 & 8.68 & 0.00 & 0.00 \\
\hline C109 & 25 & 5 & 82556.95 & 1500.00 & 82772.10 & $10800.00^{*}$ & 1500.00 & 82764.90 & 6.96 & -0.01 & 0.00 \\
\hline C201 & 25 & 5 & 186314.82 & 2000.00 & 187237.51 & 1.13 & 2000.00 & 187237.51 & 11.31 & 0.00 & 0.00 \\
\hline C202 & 25 & 5 & 186314.82 & 2000.00 & 187231.15 & 217.90 & 2000.00 & 187231.15 & 9.45 & 0.00 & 0.00 \\
\hline C203 & 25 & 5 & 186314.82 & 2000.00 & 187231.15 & $10800.00^{*}$ & 2000.00 & 187231.15 & 7.06 & 0.00 & 0.00 \\
\hline C204 & 25 & 5 & 186314.82 & 2000.00 & 187232.33 & $10800.00^{*}$ & 2000.00 & 187232.33 & 10.29 & 0.00 & 0.00 \\
\hline C205 & 25 & 5 & 186314.82 & 2000.00 & 187237.51 & 25.17 & 2000.00 & 187237.51 & 6.24 & 0.00 & 0.00 \\
\hline C206 & 25 & 5 & 186314.82 & 2000.00 & 187237.51 & 333.59 & 2000.00 & 187237.51 & 8.77 & 0.00 & 0.00 \\
\hline C207 & 25 & 5 & 186314.82 & 2000.00 & 187233.45 & 248.47 & 2000.00 & 187233.45 & 7.80 & 0.00 & 0.00 \\
\hline C208 & 25 & 5 & 186314.82 & 2000.00 & 187237.35 & 926.44 & 2000.00 & 187237.35 & 6.71 & 0.00 & 0.00 \\
\hline R101 & 25 & 5 & 37930.66 & 580.00 & 39242.52 & 8.97 & 580.00 & 39242.52 & 8.24 & 0.00 & 0.00 \\
\hline R102 & 25 & 5 & 37930.66 & 620.00 & 39163.49 & $10800.00^{*}$ & 620.00 & 39153.10 & 8.13 & -0.03 & 0.00 \\
\hline R103 & 25 & 5 & 37930.66 & 640.00 & 38144.61 & $10800.00^{*}$ & 640.00 & 38126.50 & 10.35 & -0.05 & 0.00 \\
\hline R104 & 25 & 5 & 37930.66 & 640.00 & 38111.15 & $10800.00^{*}$ & 640.00 & 38074.50 & 9.99 & -0.10 & 0.00 \\
\hline R105 & 25 & 5 & 37930.66 & 600.00 & 38225.10 & 999.73 & 600.00 & 38225.10 & 10.69 & 0.00 & 0.00 \\
\hline R106 & 25 & 5 & 37930.66 & 630.00 & 38168.91 & $10800.00^{*}$ & 630.00 & 38151.70 & 7.54 & -0.05 & 0.00 \\
\hline R107 & 25 & 5 & 37930.66 & 580.00 & 38075.13 & 10775.00 & 580.00 & 38069.40 & 11.55 & -0.02 & 0.00 \\
\hline R108 & 25 & 5 & 37930.66 & 580.00 & 38040.62 & $10800.00^{*}$ & 580.00 & 38040.62 & 6.54 & 0.00 & 0.00 \\
\hline R109 & 25 & 5 & 37930.66 & 600.00 & 38150.69 & $10800.00^{*}$ & 600.00 & 38154.90 & 8.60 & 0.01 & 0.00 \\
\hline R110 & 25 & 5 & 37930.66 & 640.00 & 38114.13 & $10800.00^{*}$ & 640.00 & 38100.50 & 10.30 & -0.04 & 0.00 \\
\hline R111 & 25 & 5 & 37930.66 & 610.00 & 38126.42 & $10800.00^{*}$ & 610.00 & 38058.30 & 6.39 & -0.18 & 0.00 \\
\hline R112 & 25 & 5 & 37930.66 & 640.00 & 38113.11 & $10800.00^{*}$ & 640.00 & 38049.50 & 9.73 & -0.17 & 0.00 \\
\hline R201 & 25 & 5 & 174795.80 & 900.00 & 175439.55 & 32.28 & 900.00 & 175439.55 & 9.61 & 0.00 & 0.00 \\
\hline R202 & 25 & 5 & 174795.80 & 900.00 & 175361.14 & $10800.00^{*}$ & 900.00 & 175361.14 & 7.58 & 0.00 & 0.00 \\
\hline R203 & 25 & 5 & 174795.80 & 900.00 & 175331.29 & 10775.00 & 900.00 & 175325.00 & 7.00 & 0.00 & 0.00 \\
\hline R204 & 25 & 5 & 174795.80 & 900.00 & 175267.13 & $10800.00^{*}$ & 900.00 & 175267.13 & 9.10 & 0.00 & 0.00 \\
\hline R205 & 25 & 5 & 174795.80 & 900.00 & 175319.60 & 567.39 & 900.00 & 175319.60 & 8.53 & 0.00 & 0.00 \\
\hline R206 & 25 & 5 & 174795.80 & 900.00 & 175294.33 & $10800.00^{*}$ & 900.00 & 175294.33 & 10.14 & 0.00 & 0.00 \\
\hline R207 & 25 & 5 & 174795.80 & 900.00 & 175295.41 & $10800.00^{*}$ & 900.00 & 175284.00 & 10.34 & -0.01 & 0.00 \\
\hline R208 & 25 & 5 & 174795.80 & 900.00 & 175256.29 & 1588.22 & 900.00 & 175256.29 & 7.30 & 0.00 & 0.00 \\
\hline R209 & 25 & 5 & 174795.80 & 900.00 & 175296.89 & 5431.05 & 900.00 & 175296.89 & 8.43 & 0.00 & 0.00 \\
\hline R210 & 25 & 5 & 174795.80 & 900.00 & 175326.94 & $10800.00^{*}$ & 900.00 & 175320.00 & 11.40 & 0.00 & 0.00 \\
\hline R211 & 25 & 5 & 174795.80 & 900.00 & 175264.11 & $10800.00^{*}$ & 900.00 & 175264.11 & 9.13 & 0.00 & 0.00 \\
\hline RC101 & 25 & 5 & 36252.02 & 990.00 & 36450.48 & 974.29 & 990.00 & 36484.40 & 8.09 & 0.09 & 0.00 \\
\hline RC102 & 25 & 5 & 36252.02 & 990.00 & 36432.10 & $10800.00^{*}$ & 990.00 & 36447.40 & 8.48 & 0.04 & 0.00 \\
\hline RC103 & 25 & 5 & 36252.02 & 990.00 & 36381.15 & $10800.00^{*}$ & 990.00 & 36403.40 & 11.18 & 0.06 & 0.00 \\
\hline RC104 & 25 & 5 & 36252.02 & 990.00 & 36378.72 & $10800.00^{*}$ & 990.00 & 36418.00 & 8.54 & 0.11 & 0.00 \\
\hline RC105 & 25 & 5 & 36252.02 & 990.00 & 36468.03 & $10800.00^{*}$ & 990.00 & 36489.50 & 10.76 & 0.06 & 0.00 \\
\hline RC106 & 25 & 5 & 36252.02 & 990.00 & 36413.53 & $10800.00^{*}$ & 990.00 & 36415.20 & 8.21 & 0.00 & 0.00 \\
\hline RC107 & 25 & 5 & 36252.02 & 990.00 & 36381.34 & $10800.00^{*}$ & 990.00 & 36392.40 & 9.16 & 0.03 & 0.00 \\
\hline RC108 & 25 & 5 & 36252.02 & 990.00 & 36409.38 & $10800.00^{*}$ & 990.00 & 36391.10 & 7.79 & -0.05 & 0.00 \\
\hline RC201 & 25 & 5 & 174075.96 & 1000.00 & 174326.81 & $10800.00^{*}$ & 1000.00 & 174326.81 & 7.98 & 0.00 & 0.00 \\
\hline RC202 & 25 & 5 & 174075.96 & 1000.00 & 174319.65 & $10800.00^{*}$ & 1000.00 & 174319.65 & 6.77 & 0.00 & 0.00 \\
\hline RC203 & 25 & 5 & 174075.96 & 1000.00 & 174309.48 & $10800.00^{*}$ & 1000.00 & 174309.48 & 11.03 & 0.00 & 0.00 \\
\hline RC204 & 25 & 5 & 174075.96 & 1000.00 & 174285.70 & $10800.00^{*}$ & 1000.00 & 174281.00 & 6.32 & 0.00 & 0.00 \\
\hline RC205 & 25 & 5 & 174075.96 & 1000.00 & 174318.88 & $10800.00^{*}$ & 1000.00 & 174318.88 & 9.16 & 0.00 & 0.00 \\
\hline RC206 & 25 & 5 & 174075.96 & 1000.00 & 174295.72 & $10800.00^{*}$ & 1000.00 & 174295.72 & 11.03 & 0.00 & 0.00 \\
\hline RC207 & 25 & 5 & 174075.96 & 1000.00 & 174280.19 & $10800.00^{*}$ & 1000.00 & 174278.00 & 11.92 & 0.00 & 0.00 \\
\hline RC208 & 25 & 5 & 174075.96 & 1000.00 & 174275.82 & $10800.00^{*}$ & 1000.00 & 174275.82 & 6.33 & 0.00 & 0.00 \\
\hline
\end{tabular}


Table A.6: Results on the 30-customer instances

\begin{tabular}{|c|c|c|c|c|c|c|c|c|c|c|c|}
\hline \multirow[t]{2}{*}{ Instance set } & \multirow[t]{2}{*}{$\left|\mathcal{N}_{c}\right|$} & \multirow[t]{2}{*}{$\left|\mathcal{N}_{0}\right|$} & \multicolumn{4}{|l|}{$E_{4}^{v}$} & \multicolumn{5}{|l|}{ HESA } \\
\hline & & & LP relaxation & Vehicle cost & Total cost & Time (s.) & Vehicle cost & Total cost & Time (s.) & $\operatorname{Dev}_{T C}$ & $\overline{\operatorname{Dev}}_{V C}$ \\
\hline C101 & 30 & 5 & 82758.85 & 1800.00 & 83097.51 & $10800.00^{*}$ & 1800.00 & 83097.50 & 14.16 & 0.00 & 0.00 \\
\hline C102 & 30 & 5 & 82758.85 & 1800.00 & 83097.51 & 505.56 & 1800.00 & 83109.40 & 17.76 & 0.01 & 0.00 \\
\hline C103 & 30 & 5 & 82758.85 & 1800.00 & 83098.28 & $10800.00^{*}$ & 1800.00 & 83109.40 & 12.33 & 0.01 & 0.00 \\
\hline C104 & 30 & 5 & 82758.85 & 1800.00 & 83094.88 & $10800.00^{*}$ & 1800.00 & 83229.20 & 15.51 & 0.16 & 0.00 \\
\hline C105 & 30 & 5 & 82758.85 & 1800.00 & 83097.51 & $10800.00^{*}$ & 1800.00 & 83097.51 & 14.21 & 0.00 & 0.00 \\
\hline C106 & 30 & 5 & 82758.85 & 1800.00 & 83097.51 & $10800.00^{*}$ & 1800.00 & 83097.51 & 15.38 & 0.00 & 0.00 \\
\hline C107 & 30 & 5 & 82758.85 & 1800.00 & 83094.88 & $10800.00^{*}$ & 1800.00 & 83094.88 & 10.70 & 0.00 & 0.00 \\
\hline C108 & 30 & 5 & 82758.85 & 1800.00 & 83094.88 & $10800.00^{*}$ & 1800.00 & 83100.60 & 11.04 & 0.01 & 0.00 \\
\hline C109 & 30 & 5 & 82758.85 & 1800.00 & 83094.88 & $10800.00^{*}$ & 1800.00 & 83101.20 & 13.48 & 0.01 & 0.00 \\
\hline C201 & 30 & 5 & 186488.20 & 2000.00 & 187255.37 & 1.86 & 2000.00 & 187255.37 & 11.09 & 0.00 & 0.00 \\
\hline C202 & 30 & 5 & 186488.20 & 2000.00 & 187249.00 & $10800.00^{*}$ & 2000.00 & 187249.00 & 11.17 & 0.00 & 0.00 \\
\hline C203 & 30 & 5 & 186488.20 & 2000.00 & 187263.79 & $10800.00^{*}$ & 2000.00 & 187249.00 & 11.56 & -0.01 & 0.00 \\
\hline C204 & 30 & 5 & 186488.20 & 2000.00 & 187245.82 & $10800.00^{*}$ & 2000.00 & 187245.82 & 10.66 & 0.00 & 0.00 \\
\hline C205 & 30 & 5 & 186488.20 & 2000.00 & 187255.37 & 34.37 & 2000.00 & 187255.37 & 12.78 & 0.00 & 0.00 \\
\hline C206 & 30 & 5 & 186488.20 & 2000.00 & 187255.37 & 543.48 & 2000.00 & 187255.37 & 16.63 & 0.00 & 0.00 \\
\hline C207 & 30 & 5 & 186488.20 & 2000.00 & 187251.31 & 377.96 & 2000.00 & 187251.31 & 13.87 & 0.00 & 0.00 \\
\hline C208 & 30 & 5 & 186488.20 & 2000.00 & 187255.56 & $10800.00^{*}$ & 2000.00 & 187255.56 & 12.75 & 0.00 & 0.00 \\
\hline R101 & 30 & 5 & 38126.33 & 710.00 & 39421.28 & 33.74 & 710.00 & 39421.28 & 10.72 & 0.00 & 0.00 \\
\hline R102 & 30 & 5 & 38126.33 & 730.00 & 39356.38 & $10800.00^{*}$ & 730.00 & 39341.30 & 10.62 & -0.04 & 0.00 \\
\hline R103 & 30 & 5 & 38126.33 & 750.00 & 38346.59 & $10800.00^{*}$ & 750.00 & 38330.30 & 13.11 & -0.04 & 0.00 \\
\hline R104 & 30 & 5 & 38126.33 & 780.00 & 38337.89 & $10800.00^{*}$ & 780.00 & 38278.40 & 10.11 & -0.16 & 0.00 \\
\hline R105 & 30 & 5 & 38126.33 & 730.00 & 38451.92 & $10800.00^{*}$ & 730.00 & 38442.10 & 10.83 & -0.03 & 0.00 \\
\hline R106 & 30 & 5 & 38126.33 & 790.00 & 38457.92 & $10800.00^{*}$ & 790.00 & 38353.70 & 10.77 & -0.27 & 0.00 \\
\hline R107 & 30 & 5 & 38126.33 & 780.00 & 38357.13 & $10800.00^{*}$ & 780.00 & 38295.40 & 12.79 & -0.16 & 0.00 \\
\hline R108 & 30 & 5 & 38126.33 & 780.00 & 38326.78 & $10800.00^{*}$ & 780.00 & 38257.70 & 11.83 & -0.18 & 0.00 \\
\hline R109 & 30 & 5 & 38126.33 & 780.00 & 38396.41 & $10800.00^{*}$ & 780.00 & 38355.90 & 10.24 & -0.11 & 0.00 \\
\hline R110 & 30 & 5 & 38126.33 & 780.00 & 38351.22 & $10800.00^{*}$ & 780.00 & 38321.90 & 13.68 & -0.08 & 0.00 \\
\hline R111 & 30 & 5 & 38126.33 & 780.00 & 38363.48 & $10800.00^{*}$ & 780.00 & 38285.10 & 15.04 & -0.20 & 0.00 \\
\hline R112 & 30 & 5 & 38126.33 & 780.00 & 38350.33 & $10800.00^{*}$ & 780.00 & 38265.00 & 11.21 & -0.22 & 0.00 \\
\hline R201 & 30 & 5 & 174930.96 & 900.00 & 175537.23 & 628.20 & 900.00 & 175542.00 & 13.23 & 0.00 & 0.00 \\
\hline R202 & 30 & 5 & 174930.96 & 900.00 & 175502.53 & $10800.00^{*}$ & 900.00 & 175443.00 & 15.93 & -0.03 & 0.00 \\
\hline R203 & 30 & 5 & 174930.96 & 900.00 & 175386.34 & $10800.00^{*}$ & 900.00 & 175360.00 & 11.43 & -0.02 & 0.00 \\
\hline R204 & 30 & 5 & 174930.96 & 900.00 & 175283.96 & $10800.00^{*}$ & 900.00 & 175283.96 & 11.37 & 0.00 & 0.00 \\
\hline R205 & 30 & 5 & 174930.96 & 900.00 & 175382.37 & 6435.25 & 900.00 & 175382.37 & 12.45 & 0.00 & 0.00 \\
\hline R206 & 30 & 5 & 174930.96 & 900.00 & 175370.08 & $10800.00^{*}$ & 900.00 & 175344.00 & 15.26 & -0.01 & 0.00 \\
\hline R207 & 30 & 5 & 174930.96 & 900.00 & 175327.23 & $10800.00^{*}$ & 900.00 & 175303.00 & 14.98 & -0.01 & 0.00 \\
\hline R208 & 30 & 5 & 174930.96 & 900.00 & 175283.11 & $10800.00^{*}$ & 900.00 & 175273.00 & 14.42 & -0.01 & 0.00 \\
\hline R209 & 30 & 5 & 174930.96 & 900.00 & 175338.33 & $10800.00^{*}$ & 900.00 & 175338.33 & 15.22 & 0.00 & 0.00 \\
\hline R210 & 30 & 5 & 174930.96 & 900.00 & 175353.04 & $10800.00^{*}$ & 900.00 & 175353.04 & 15.09 & 0.00 & 0.00 \\
\hline R211 & 30 & 5 & 174930.96 & 900.00 & 175323.38 & $10800.00^{*}$ & 900.00 & 175298.00 & 18.00 & -0.01 & 0.00 \\
\hline RC101 & 30 & 5 & 36474.96 & 1080.00 & 36803.52 & $10800.00^{*}$ & 1080.00 & 36814.10 & 14.93 & 0.03 & 0.00 \\
\hline RC102 & 30 & 5 & 36474.96 & 1110.00 & 36766.79 & $10800.00^{*}$ & 1110.00 & 36786.90 & 13.19 & 0.05 & 0.00 \\
\hline RC103 & 30 & 5 & 36474.96 & 1140.00 & 36696.26 & $10800.00^{*}$ & 1140.00 & 36684.60 & 13.31 & -0.03 & 0.00 \\
\hline RC104 & 30 & 5 & 36474.96 & 1140.00 & 36667.39 & $10800.00^{*}$ & 1140.00 & 36697.90 & 13.36 & 0.08 & 0.00 \\
\hline RC105 & 30 & 5 & 36474.96 & 1200.00 & 36746.25 & $10800.00^{*}$ & 1200.00 & 36748.20 & 10.20 & 0.01 & 0.00 \\
\hline RC106 & 30 & 5 & 36474.96 & 1140.00 & 36688.56 & $10800.00^{*}$ & 1140.00 & 36688.56 & 11.40 & 0.00 & 0.00 \\
\hline RC107 & 30 & 5 & 36474.96 & 1110.00 & 36699.96 & $10800.00^{*}$ & 1110.00 & 36664.60 & 13.51 & -0.10 & 0.00 \\
\hline RC108 & 30 & 5 & 36474.96 & 1140.00 & 36657.64 & $10800.00^{*}$ & 1140.00 & 36655.10 & 11.61 & -0.01 & 0.00 \\
\hline RC201 & 30 & 5 & 36199.06 & 1100.00 & 36585.08 & $10800.00^{*}$ & 1100.00 & 36578.50 & 13.03 & -0.02 & 0.00 \\
\hline RC202 & 30 & 5 & 36199.06 & 1100.00 & 36543.55 & $10800.00^{*}$ & 1100.00 & 36539.90 & 13.79 & -0.01 & 0.00 \\
\hline RC203 & 30 & 5 & 36199.06 & 1100.00 & 36540.45 & $10800.00^{*}$ & 1100.00 & 36606.90 & 10.67 & 0.18 & 0.00 \\
\hline RC204 & 30 & 5 & 36199.06 & 1100.00 & 36565.95 & $10800.00^{*}$ & 1100.00 & 36556.30 & 12.14 & -0.03 & 0.00 \\
\hline RC205 & 30 & 5 & 36199.06 & 1150.00 & 36548.96 & $10800.00^{*}$ & 1150.00 & 36548.96 & 15.53 & 0.00 & 0.00 \\
\hline RC206 & 30 & 5 & 36199.06 & 1150.00 & 36554.74 & $10800.00^{*}$ & 1150.00 & 36584.10 & 14.00 & 0.08 & 0.00 \\
\hline RC207 & 30 & 5 & 36199.06 & 1150.00 & 36528.09 & $10800.00^{*}$ & 1150.00 & 36528.09 & 11.64 & 0.00 & 0.00 \\
\hline RC208 & 30 & 5 & 36199.06 & 1200.00 & 36577.81 & $10800.00^{*}$ & 1200.00 & 36635.10 & 14.66 & 0.16 & 0.00 \\
\hline
\end{tabular}


Table A.7: Results on the 50-customer instances

\begin{tabular}{|c|c|c|c|c|c|c|c|c|c|c|c|}
\hline \multirow[t]{2}{*}{ Instance set } & \multirow[t]{2}{*}{$\left|\mathcal{N}_{c}\right|$} & \multirow[t]{2}{*}{$\left|\mathcal{N}_{0}\right|$} & \multicolumn{4}{|l|}{$E_{4}^{v}$} & \multicolumn{5}{|l|}{ HESA } \\
\hline & & & LP relaxation & Vehicle cost & Total cost & Time (s.) & Vehicle cost & Total cost & Time (s.) & $\operatorname{Dev}_{T C}$ & $\overline{\operatorname{Dev}}_{V C}$ \\
\hline C101 & 50 & 7 & 41386.56 & 2700.00 & 41865.26 & 398.97 & 2700.00 & 41892.30 & 70.96 & 0.06 & 0.00 \\
\hline C102 & 50 & 7 & 41386.56 & 2700.00 & 41859.80 & $10800.00^{*}$ & 2700.00 & 41859.80 & 103.47 & 0.00 & 0.00 \\
\hline C103 & 50 & 7 & 41386.56 & 2900.00 & 41968.98 & $10800.00^{*}$ & 2900.00 & 41922.80 & 61.00 & -0.11 & 0.00 \\
\hline C104 & 50 & 7 & 41386.56 & 2700.00 & 41890.98 & $10800.00^{*}$ & 2700.00 & 42000.50 & 114.55 & 0.26 & 0.00 \\
\hline C105 & 50 & 7 & 41386.56 & 2700.00 & 41852.69 & $10800.00^{*}$ & 2700.00 & 41852.69 & 81.52 & 0.00 & 0.00 \\
\hline C106 & 50 & 7 & 41386.56 & 2700.00 & 41853.79 & $10800.00^{*}$ & 2700.00 & 41853.79 & 95.53 & 0.00 & 0.00 \\
\hline C107 & 50 & 7 & 41386.56 & 2700.00 & 41851.28 & $10800.00^{*}$ & 2700.00 & 41903.50 & 106.71 & 0.12 & 0.00 \\
\hline C108 & 50 & 7 & 41386.56 & 2700.00 & 41850.25 & $10800.00^{*}$ & 2700.00 & 41895.50 & 105.26 & 0.11 & 0.00 \\
\hline C109 & 50 & 7 & 41386.56 & 2700.00 & 41890.31 & $10800.00^{*}$ & 2700.00 & 42016.20 & 98.83 & 0.30 & 0.00 \\
\hline C201 & 50 & 7 & 92446.10 & 2400.00 & 92880.93 & 6.21 & 2400.00 & 92880.93 & 109.43 & 0.00 & 0.00 \\
\hline C202 & 50 & 7 & 92446.10 & 3000.00 & 93384.81 & $10800.00^{*}$ & 2400.00 & 92825.00 & 62.39 & -0.60 & -20.00 \\
\hline C203 & 50 & 7 & 92446.10 & 3400.00 & 93869.63 & $10800.00^{*}$ & 2400.00 & 92818.60 & 118.84 & -1.12 & -29.41 \\
\hline C204 & 50 & 7 & 92446.10 & 3000.00 & 93470.84 & $10800.00^{*}$ & 2400.00 & 92770.40 & 115.62 & -0.75 & -20.00 \\
\hline C205 & 50 & 7 & 92446.10 & 3000.00 & 93391.05 & $10800.00^{*}$ & 2400.00 & 92876.60 & 115.28 & -0.55 & -20.00 \\
\hline C206 & 50 & 7 & 92446.10 & 3000.00 & 93391.05 & $10800.00^{*}$ & 2400.00 & 92811.40 & 66.99 & -0.62 & -20.00 \\
\hline C207 & 50 & 7 & 92446.10 & 3000.00 & 93378.45 & $10800.00^{*}$ & 2400.00 & 92798.30 & 117.47 & -0.62 & -20.00 \\
\hline C208 & 50 & 7 & 92446.10 & 3000.00 & 93390.88 & $10800.00^{*}$ & 2400.00 & 92798.40 & 87.68 & -0.63 & -20.00 \\
\hline R101 & 50 & 7 & 38870.22 & 1280.00 & 40443.15 & $10800.00^{*}$ & 1250.00 & 40443.15 & 83.30 & 0.00 & -2.34 \\
\hline R102 & 50 & 7 & 38870.22 & 1380.00 & 40440.99 & $10800.00^{*}$ & 1350.00 & 40328.30 & 95.11 & -0.28 & -2.17 \\
\hline R103 & 50 & 7 & 38870.22 & 1430.00 & 41424.71 & $10800.00^{*}$ & 1280.00 & 40195.20 & 83.30 & -2.97 & -10.49 \\
\hline R104 & 50 & 7 & 38870.22 & 1360.00 & 39368.54 & $10800.00^{*}$ & 1370.00 & 39155.50 & 112.85 & -0.54 & 0.74 \\
\hline R105 & 50 & 7 & 38870.22 & 1280.00 & 39380.48 & $10800.00^{*}$ & 1260.00 & 39356.20 & 113.54 & -0.06 & -1.56 \\
\hline R106 & 50 & 7 & 38870.22 & 1320.00 & 39459.31 & $10800.00^{*}$ & 1280.00 & 39263.10 & 119.24 & -0.50 & -3.03 \\
\hline R107 & 50 & 7 & 38870.22 & 1430.00 & 39443.09 & $10800.00^{*}$ & 1310.00 & 39152.30 & 92.82 & -0.74 & -8.39 \\
\hline R108 & 50 & 7 & 38870.22 & 1300.00 & 39322.58 & $10800.00^{*}$ & 1370.00 & 39157.10 & 104.15 & -0.42 & 5.38 \\
\hline R109 & 50 & 7 & 38870.22 & 1350.00 & 39322.03 & $10800.00^{*}$ & 1280.00 & 39250.70 & 72.21 & -0.18 & -5.19 \\
\hline R110 & 50 & 7 & 38870.22 & 1320.00 & 39411.26 & $10800.00^{*}$ & 1310.00 & 39211.80 & 61.42 & -0.51 & -0.76 \\
\hline R111 & 50 & 7 & 38870.22 & 1300.00 & 39255.20 & $10800.00^{*}$ & 1280.00 & 39179.70 & 84.72 & -0.19 & -1.54 \\
\hline R112 & 50 & 7 & 38870.22 & 1450.00 & 39403.04 & $10800.00^{*}$ & 1280.00 & 39170.50 & 97.18 & -0.59 & -11.72 \\
\hline R201 & 50 & 7 & 175518.42 & 1350.00 & 176218.56 & $10800.00^{*}$ & 1350.00 & 176218.56 & 86.87 & 0.00 & 0.00 \\
\hline R202 & 50 & 7 & 175518.42 & 2050.00 & 177013.02 & $10800.00^{*}$ & 1350.00 & 176088.00 & 91.59 & -0.52 & -34.15 \\
\hline R203 & 50 & 7 & 175518.42 & 1350.00 & 176249.23 & $10800.00^{*}$ & 1350.00 & 175995.00 & 93.72 & -0.14 & 0.00 \\
\hline R204 & 50 & 7 & 175518.42 & 1350.00 & 175917.21 & $10800.00^{*}$ & 1350.00 & 175866.00 & 118.70 & -0.03 & 0.00 \\
\hline R205 & 50 & 7 & 175518.42 & 1350.00 & 176200.21 & $10800.00^{*}$ & 1350.00 & 176061.00 & 90.21 & -0.08 & 0.00 \\
\hline R206 & 50 & 7 & 175518.42 & 1350.00 & 176081.07 & $10800.00^{*}$ & 1350.00 & 176000.00 & 117.02 & -0.05 & 0.00 \\
\hline R207 & 50 & 7 & 175518.42 & 2050.00 & 176815.10 & $10800.00^{*}$ & 1350.00 & 175932.00 & 119.13 & -0.50 & -34.15 \\
\hline R208 & 50 & 7 & 175518.42 & 1350.00 & 175962.50 & $10800.00^{*}$ & 1350.00 & 175855.00 & 63.31 & -0.06 & 0.00 \\
\hline R209 & 50 & 7 & 175518.42 & 1350.00 & 176089.81 & $10800.00^{*}$ & 1350.00 & 175995.00 & 69.61 & -0.05 & 0.00 \\
\hline R210 & 50 & 7 & 175518.42 & 1600.00 & 176374.18 & $10800.00^{*}$ & 1350.00 & 175994.00 & 82.15 & -0.22 & -15.63 \\
\hline R211 & 50 & 7 & 175518.42 & 1800.00 & 176704.38 & $10800.00^{*}$ & 1350.00 & 175905.00 & 87.41 & -0.45 & -25.00 \\
\hline RC101 & 50 & 7 & 19574.34 & 1950.00 & 21031.12 & $10800.00^{*}$ & 1860.00 & 20978.50 & 83.08 & -0.25 & -4.62 \\
\hline RC102 & 50 & 7 & 19574.34 & 2280.00 & 21334.83 & $10800.00^{*}$ & 1950.00 & 20892.30 & 63.81 & -2.07 & -14.47 \\
\hline RC103 & 50 & 7 & 19574.34 & 2130.00 & 38142.81 & $10800.00^{*}$ & 1950.00 & 20778.80 & 74.35 & -45.52 & -8.45 \\
\hline RC104 & 50 & 7 & 19574.34 & 2250.00 & 20274.62 & $10800.00^{*}$ & 2010.00 & 20020.00 & 105.47 & -1.26 & -10.67 \\
\hline RC105 & 50 & 7 & 19574.34 & 2010.00 & 20991.89 & $10800.00^{*}$ & 1950.00 & 20889.10 & 72.03 & -0.49 & -2.99 \\
\hline RC106 & 50 & 7 & 19574.34 & 2040.00 & 20325.56 & $10800.00^{*}$ & 1950.00 & 20223.20 & 76.47 & -0.50 & -4.41 \\
\hline RC107 & 50 & 7 & 19574.34 & 2040.00 & 20291.25 & $10800.00^{*}$ & 1860.00 & 20085.60 & 61.74 & -1.01 & -8.82 \\
\hline RC108 & 50 & 7 & 19574.34 & 1950.00 & 20064.39 & $10800.00^{*}$ & 1980.00 & 20018.40 & 75.42 & -0.23 & 1.54 \\
\hline RC201 & 50 & 7 & 87065.42 & 1700.00 & 87586.41 & $10800.00^{*}$ & 1800.00 & 87641.90 & 94.32 & 0.06 & 5.88 \\
\hline RC202 & 50 & 7 & 87065.42 & 1900.00 & 87992.95 & $10800.00^{*}$ & 1800.00 & 87546.10 & 65.43 & -0.51 & -5.26 \\
\hline RC203 & 50 & 7 & 87065.42 & 2250.00 & 88339.08 & $10800.00^{*}$ & 1900.00 & 87558.60 & 114.14 & -0.88 & -15.56 \\
\hline RC204 & 50 & 7 & 87065.42 & 3550.00 & 89274.98 & $10800.00^{*}$ & 1900.00 & 87456.90 & 61.20 & -2.04 & -46.48 \\
\hline RC205 & 50 & 7 & 87065.42 & 1900.00 & 87784.60 & $10800.00^{*}$ & 1800.00 & 87530.90 & 76.40 & -0.29 & -5.26 \\
\hline RC206 & 50 & 7 & 87065.42 & 1750.00 & 87989.08 & $10800.00^{*}$ & 1900.00 & 87619.80 & 69.17 & -0.42 & 8.57 \\
\hline RC207 & 50 & 7 & 87065.42 & 1850.00 & 88092.32 & $10800.00^{*}$ & 1900.00 & 87469.30 & 104.49 & -0.71 & 2.70 \\
\hline RC208 & 50 & 7 & 87065.42 & 2550.00 & 88410.89 & $10800.00^{*}$ & 1950.00 & 87464.80 & 99.78 & -1.07 & -23.53 \\
\hline
\end{tabular}


Table A.8: Results on the 75-customer instances

\begin{tabular}{|c|c|c|c|c|c|c|c|c|c|c|c|}
\hline \multirow[t]{2}{*}{ Instance set } & \multirow[t]{2}{*}{$\left|\mathcal{N}_{c}\right|$} & \multirow[t]{2}{*}{$\left|\mathcal{N}_{0}\right|$} & \multicolumn{4}{|l|}{$E_{4}^{v}$} & \multicolumn{5}{|l|}{ HESA } \\
\hline & & & LP relaxation & Vehicle cost & Total cost & Time (s.) & Vehicle cost & Total cost & Time (s.) & $\operatorname{Dev}_{T C}$ & $\overline{\operatorname{Dev}}_{V C}$ \\
\hline C101 & 75 & 8 & 82857.15 & 4200.00 & 83309.83 & $10800.00^{*}$ & 4200.00 & 83376.60 & 187.36 & 0.08 & 0.00 \\
\hline C102 & 75 & 8 & 82857.15 & 4200.00 & 83343.28 & $10800.00^{*}$ & 4200.00 & 83389.00 & 185.65 & 0.05 & 0.00 \\
\hline C103 & 75 & 8 & 82857.15 & 5350.00 & 84468.47 & $10800.00^{*}$ & 4200.00 & 83321.10 & 157.32 & -1.36 & -21.50 \\
\hline C104 & 75 & 8 & 82857.15 & 5450.00 & 84670.05 & $10800.00^{*}$ & 4800.00 & 83766.60 & 159.44 & -1.07 & -11.93 \\
\hline C105 & 75 & 8 & 82857.15 & 4200.00 & 83311.92 & $10800.00^{*}$ & 4200.00 & 83489.80 & 164.07 & 0.21 & 0.00 \\
\hline C106 & 75 & 8 & 82857.15 & 4200.00 & 83307.33 & $10800.00^{*}$ & 4200.00 & 83372.90 & 184.56 & 0.08 & 0.00 \\
\hline C107 & 75 & 8 & 82857.15 & 4200.00 & 83308.44 & $10800.00^{*}$ & 4200.00 & 83632.90 & 179.85 & 0.39 & 0.00 \\
\hline C108 & 75 & 8 & 82857.15 & 4200.00 & 83450.51 & $10800.00^{*}$ & 4200.00 & 83411.10 & 176.41 & -0.05 & 0.00 \\
\hline C109 & 75 & 8 & 82857.15 & 4600.00 & 83792.90 & $10800.00^{*}$ & 4400.00 & 83555.50 & 165.48 & -0.28 & -4.35 \\
\hline C201 & 75 & 8 & 188792.53 & 4000.00 & 189585.40 & $10800.00^{*}$ & 3800.00 & 189401.00 & 163.23 & -0.10 & -5.00 \\
\hline C202 & 75 & 8 & 188792.53 & 6400.00 & 192152.42 & $10800.00^{*}$ & 3800.00 & 189371.00 & 161.08 & -1.45 & -40.63 \\
\hline C203 & 75 & 8 & 188792.53 & 7500.00 & 193453.62 & $10800.00^{*}$ & 3800.00 & 189360.00 & 174.90 & -2.12 & -49.33 \\
\hline C204 & 75 & 8 & 188792.53 & 13800.00 & 200531.39 & $10800.00^{*}$ & 3800.00 & 189328.00 & 141.79 & -5.59 & -72.46 \\
\hline C205 & 75 & 8 & 188792.53 & 4000.00 & 189582.69 & $10800.00^{*}$ & 3800.00 & 189400.00 & 144.57 & -0.10 & -5.00 \\
\hline C206 & 75 & 8 & 188792.53 & 5000.00 & 190731.82 & $10800.00^{*}$ & 3800.00 & 189376.00 & 180.62 & -0.71 & -24.00 \\
\hline C207 & 75 & 8 & 188792.53 & 7100.00 & 193275.65 & $10800.00^{*}$ & 3800.00 & 189390.00 & 170.03 & -2.01 & -46.48 \\
\hline C208 & 75 & 8 & 188792.53 & 8800.00 & 194531.18 & $10800.00^{*}$ & 3800.00 & 189372.00 & 186.91 & -2.65 & -56.82 \\
\hline R101 & 75 & 8 & 38056.29 & 1970.00 & 40600.20 & $10800.00^{*}$ & 1880.00 & 40609.90 & 189.28 & 0.02 & -4.57 \\
\hline R102 & 75 & 8 & 38056.29 & 2040.00 & 40913.94 & $10800.00^{*}$ & 1900.00 & 40437.50 & 162.13 & -1.16 & -6.86 \\
\hline R103 & 75 & 8 & 38056.29 & 8370.00 & 176468.82 & $10800.00^{*}$ & 1960.00 & 40252.60 & 147.57 & -77.19 & -76.58 \\
\hline R104 & 75 & 8 & 38056.29 & 3290.00 & 40584.42 & $10800.00^{*}$ & 2010.00 & 40256.90 & 188.58 & -0.81 & -38.91 \\
\hline R105 & 75 & 8 & 38056.29 & 2060.00 & 40643.62 & $10800.00^{*}$ & 1920.00 & 40368.50 & 172.37 & -0.68 & -6.80 \\
\hline R106 & 75 & 8 & 38056.29 & 4140.00 & 103148.58 & $10800.00^{*}$ & 1920.00 & 40295.60 & 157.16 & -60.93 & -53.62 \\
\hline R107 & 75 & 8 & 38056.29 & 4860.00 & 81617.99 & $10800.00^{*}$ & 2010.00 & 40332.90 & 173.12 & -50.58 & -58.64 \\
\hline R108 & 75 & 8 & 38056.29 & 5390.00 & 61602.75 & $10800.00^{*}$ & 2120.00 & 40234.80 & 156.94 & -34.69 & -60.67 \\
\hline R109 & 75 & 8 & 38056.29 & 2760.00 & 39933.80 & $10800.00^{*}$ & 1980.00 & 38825.00 & 171.10 & -2.78 & -28.26 \\
\hline R110 & 75 & 8 & 38056.29 & 4660.00 & 42288.53 & $10800.00^{*}$ & 1960.00 & 38715.10 & 182.79 & -8.45 & -57.94 \\
\hline R111 & 75 & 8 & 38056.29 & 5290.00 & 61206.47 & $10800.00^{*}$ & 2010.00 & 40281.90 & 186.65 & -34.19 & -62.00 \\
\hline R112 & 75 & 8 & 38056.29 & 3670.00 & 40971.58 & $10800.00^{*}$ & 1960.00 & 38660.40 & 177.68 & -5.64 & -46.59 \\
\hline R201 & 75 & 8 & 174160.82 & 2250.00 & 175775.01 & $10800.00^{*}$ & 1800.00 & 174859.00 & 152.31 & -0.52 & -20.00 \\
\hline R202 & 75 & 8 & 174160.82 & 5900.00 & 179505.22 & $10800.00^{*}$ & 1800.00 & 174767.00 & 145.66 & -2.64 & -69.49 \\
\hline R203 & 75 & 8 & 174160.82 & 8700.00 & 182253.80 & $10800.00^{*}$ & 1800.00 & 174601.00 & 171.49 & -4.20 & -79.31 \\
\hline R204 & 75 & 8 & 174160.82 & 5000.00 & 177907.86 & $10800.00^{*}$ & 1800.00 & 174472.00 & 173.79 & -1.93 & -64.00 \\
\hline R205 & 75 & 8 & 174160.82 & 2950.00 & 176368.20 & $10800.00^{*}$ & 1800.00 & 174659.00 & 141.53 & -0.97 & -38.98 \\
\hline R206 & 75 & 8 & 174160.82 & 3700.00 & 176973.52 & $10800.00^{*}$ & 1800.00 & 174597.00 & 178.87 & -1.34 & -51.35 \\
\hline R207 & 75 & 8 & 174160.82 & 4650.00 & 178021.12 & $10800.00^{*}$ & 1800.00 & 174524.00 & 173.78 & -1.96 & -61.29 \\
\hline R208 & 75 & 8 & 174160.82 & 5750.00 & 178926.32 & $10800.00^{*}$ & 1800.00 & 174445.00 & 150.08 & -2.50 & -68.70 \\
\hline R209 & 75 & 8 & 174160.82 & 6550.00 & 179798.66 & $10800.00^{*}$ & 1800.00 & 174563.00 & 176.27 & -2.91 & -72.52 \\
\hline R210 & 75 & 8 & 174160.82 & 5700.00 & 179194.44 & $10800.00^{*}$ & 1800.00 & 174605.00 & 150.63 & -2.56 & -68.42 \\
\hline R211 & 75 & 8 & 174160.82 & 6550.00 & 179843.07 & $10800.00^{*}$ & 1800.00 & 174522.00 & 166.47 & -2.96 & -72.52 \\
\hline RC101 & 75 & 8 & 38233.86 & 2520.00 & 39061.10 & $10800.00^{*}$ & 2520.00 & 38940.60 & 172.15 & -0.31 & 0.00 \\
\hline RC102 & 75 & 8 & 38233.86 & 2640.00 & 39221.20 & $10800.00^{*}$ & 2550.00 & 38832.50 & 174.34 & -0.99 & -3.41 \\
\hline RC103 & 75 & 8 & 38233.86 & 3510.00 & 39820.43 & $10800.00^{*}$ & 2580.00 & 38717.80 & 183.92 & -2.77 & -26.50 \\
\hline RC104 & 75 & 8 & 38233.86 & 3480.00 & 39830.82 & $10800.00^{*}$ & 2700.00 & 38677.30 & 158.68 & -2.90 & -22.41 \\
\hline RC105 & 75 & 8 & 38233.86 & 2760.00 & 39258.60 & $10800.00^{*}$ & 2580.00 & 38866.70 & 167.90 & -1.00 & -6.52 \\
\hline RC106 & 75 & 8 & 38233.86 & 2910.00 & 39318.93 & $10800.00^{*}$ & 2610.00 & 38795.00 & 183.80 & -1.33 & -10.31 \\
\hline RC107 & 75 & 8 & 38233.86 & 3390.00 & 39692.26 & $10800.00^{*}$ & 2670.00 & 38676.20 & 165.33 & -2.56 & -21.24 \\
\hline RC108 & 75 & 8 & 38233.86 & 3480.00 & 39984.80 & $10800.00^{*}$ & 2550.00 & 38645.00 & 186.19 & -3.35 & -26.72 \\
\hline RC201 & 75 & 8 & 173677.24 & 2250.00 & 174872.25 & $10800.00^{*}$ & 2350.00 & 174546.00 & 159.46 & -0.19 & 4.44 \\
\hline RC202 & 75 & 8 & 173677.24 & 4700.00 & 177692.77 & $10800.00^{*}$ & 2350.00 & 174332.00 & 162.91 & -1.89 & -50.00 \\
\hline RC203 & 75 & 8 & 173677.24 & 5550.00 & 178208.59 & $10800.00^{*}$ & 2400.00 & 174373.00 & 173.16 & -2.15 & -56.76 \\
\hline RC204 & 75 & 8 & 173677.24 & 6250.00 & 179071.17 & $10800.00^{*}$ & 2550.00 & 174241.00 & 185.53 & -2.70 & -59.20 \\
\hline RC205 & 75 & 8 & 173677.24 & 2900.00 & 175681.10 & $10800.00^{*}$ & 2400.00 & 174444.00 & 152.35 & -0.70 & -17.24 \\
\hline RC206 & 75 & 8 & 173677.24 & 2650.00 & 175218.43 & $10800.00^{*}$ & 2450.00 & 174419.00 & 147.26 & -0.46 & -7.55 \\
\hline RC207 & 75 & 8 & 173677.24 & 5300.00 & 178097.38 & $10800.00^{*}$ & 2550.00 & 174346.00 & 144.99 & -2.11 & -51.89 \\
\hline RC208 & 75 & 8 & 173677.24 & 3800.00 & 176137.37 & $10800.00^{*}$ & 2550.00 & 174242.00 & 155.45 & -1.08 & -32.89 \\
\hline
\end{tabular}


Table A.9: Results on the 100-customer instances

\begin{tabular}{|c|c|c|c|c|c|c|c|c|c|c|c|}
\hline \multirow[t]{2}{*}{ Instance set } & \multirow[t]{2}{*}{$\left|\mathcal{N}_{c}\right|$} & \multirow[t]{2}{*}{$\left|\mathcal{N}_{0}\right|$} & \multicolumn{4}{|l|}{$E_{4}^{v}$} & \multicolumn{5}{|l|}{ HESA } \\
\hline & & & LP relaxation & Vehicle cost & Total cost & Time (s.) & Vehicle cost & Total cost & Time (s.) & $\operatorname{Dev}_{T C}$ & $\operatorname{Dev}_{V C}$ \\
\hline C101 & 100 & 10 & 84345.45 & 5700.00 & 85199.09 & $10800.00^{*}$ & 5700.00 & 85199.09 & 297.32 & 0.00 & 0.00 \\
\hline C102 & 100 & 10 & 84345.45 & 6300.00 & 86605.08 & $10800.00^{*}$ & 5700.00 & 85209.00 & 304.48 & -1.61 & -9.52 \\
\hline C103 & 100 & 10 & 84345.45 & 8300.00 & 88128.63 & $10800.00^{*}$ & 5700.00 & 85190.80 & 305.06 & -3.33 & -31.33 \\
\hline C104 & 100 & 10 & 84345.45 & 11000.00 & 90622.34 & $10800.00^{*}$ & 6500.00 & 85509.90 & 319.80 & -5.64 & -40.91 \\
\hline C105 & 100 & 10 & 84345.45 & 5700.00 & 85258.60 & $10800.00^{*}$ & 5700.00 & 85208.50 & 319.99 & -0.06 & 0.00 \\
\hline C106 & 100 & 10 & 84345.45 & 5700.00 & 85461.36 & $10800.00^{*}$ & 5700.00 & 85204.90 & 309.38 & -0.30 & 0.00 \\
\hline C107 & 100 & 10 & 84345.45 & 5700.00 & 85306.67 & $10800.00^{*}$ & 5700.00 & 85167.30 & 307.85 & -0.16 & 0.00 \\
\hline C108 & 100 & 10 & 84345.45 & 5900.00 & 85570.18 & $10800.00^{*}$ & 5700.00 & 85222.80 & 294.20 & -0.41 & -3.39 \\
\hline C109 & 100 & 10 & 84345.45 & 9100.00 & 89617.66 & $10800.00^{*}$ & 5700.00 & 85198.00 & 312.34 & -4.93 & -37.36 \\
\hline C201 & 100 & 10 & 190083.07 & 6000.00 & 191687.14 & $10800.00^{*}$ & 5200.00 & 190876.00 & 271.94 & -0.42 & -13.33 \\
\hline C202 & 100 & 10 & 190074.71 & 9900.00 & 195858.81 & $10800.00^{*}$ & 5200.00 & 190865.00 & 259.04 & -2.55 & -47.47 \\
\hline C203 & 100 & 10 & 190075.95 & 17900.00 & 304096.37 & $10800.00^{*}$ & 5200.00 & 190864.00 & 308.09 & -37.24 & -70.95 \\
\hline C204 & 100 & 10 & 190074.71 & 98300.00 & 1154483.31 & $10800.00^{*}$ & 5200.00 & 190848.00 & 337.23 & -83.47 & -94.71 \\
\hline C205 & 100 & 10 & 190077.15 & 8400.00 & 194711.89 & $10800.00^{*}$ & 5200.00 & 190863.00 & 304.44 & -1.98 & -38.10 \\
\hline C206 & 100 & 10 & 190084.61 & 15600.00 & 202111.00 & $10800.00^{*}$ & 5200.00 & 190861.00 & 321.63 & -5.57 & -66.67 \\
\hline C207 & 100 & 10 & 190075.31 & 13200.00 & 199617.46 & $10800.00^{*}$ & 5200.00 & 190856.00 & 330.40 & -4.39 & -60.61 \\
\hline C208 & 100 & 10 & 190074.82 & 13800.00 & 200924.51 & $10800.00^{*}$ & 5200.00 & 190866.00 & 333.46 & -5.01 & -62.32 \\
\hline R101 & 100 & 10 & 39159.76 & 2770.00 & 42095.43 & $10800.00^{*}$ & 2560.00 & 41782.20 & 292.25 & -0.74 & -7.58 \\
\hline R102 & 100 & 10 & 39146.09 & 7090.00 & 105133.04 & $10800.00^{*}$ & 2600.00 & 41577.10 & 324.69 & -60.45 & -63.33 \\
\hline R103 & 100 & 10 & 39149.89 & 10860.00 & 225357.73 & $10800.00^{*}$ & 2620.00 & 41398.40 & 293.22 & -81.63 & -75.87 \\
\hline R104 & 100 & 10 & 39151.07 & 12740.00 & 227609.71 & $10800.00^{*}$ & 2680.00 & 41269.90 & 289.51 & -81.87 & -78.96 \\
\hline R105 & 100 & 10 & 39154.42 & 4790.00 & 81860.12 & $10800.00^{*}$ & 2670.00 & 41522.20 & 250.56 & -49.28 & -44.26 \\
\hline R106 & 100 & 10 & 39145.82 & 12070.00 & 226936.85 & $10800.00^{*}$ & 2650.00 & 41397.50 & 291.75 & -81.76 & -78.04 \\
\hline R107 & 100 & 10 & 39145.98 & 14680.00 & 230534.40 & $10800.00^{*}$ & 2680.00 & 41329.90 & 339.02 & -82.07 & -81.74 \\
\hline R108 & 100 & 10 & 39148.67 & 12390.00 & 227135.02 & $10800.00^{*}$ & 2680.00 & 40574.50 & 336.96 & -82.14 & -78.37 \\
\hline R109 & 100 & 10 & 39161.60 & 8300.00 & 106140.17 & $10800.00^{*}$ & 2710.00 & 40135.40 & 247.20 & -62.19 & -67.35 \\
\hline R110 & 100 & 10 & 39146.09 & 8800.00 & 68339.42 & $10800.00^{*}$ & 2600.00 & 40052.30 & 267.95 & -41.39 & -70.45 \\
\hline R111 & 100 & 10 & 39145.98 & 12590.00 & 228378.35 & $10800.00^{*}$ & 2600.00 & 40614.10 & 296.14 & -82.22 & -79.35 \\
\hline R112 & 100 & 10 & 39165.22 & 10900.00 & 226034.92 & $10800.00^{*}$ & 2730.00 & 40015.60 & 250.16 & -82.30 & -74.95 \\
\hline R201 & 100 & 10 & 174794.14 & 6400.00 & 180128.14 & $10800.00^{*}$ & 2250.00 & 175457.00 & 329.90 & -2.59 & -64.84 \\
\hline R202 & 100 & 10 & 174794.09 & 14450.00 & 188288.16 & $10800.00^{*}$ & 2250.00 & 175303.00 & 256.58 & -6.90 & -84.43 \\
\hline R203 & 100 & 10 & 174794.09 & 12850.00 & 186985.40 & $10800.00^{*}$ & 2250.00 & 175186.00 & 289.80 & -6.31 & -82.49 \\
\hline R204 & 100 & 10 & 174794.09 & 11250.00 & 184455.99 & $10800.00^{*}$ & 2250.00 & 175037.00 & 297.46 & -5.11 & -80.00 \\
\hline R205 & 100 & 10 & 174794.09 & 12450.00 & 186185.59 & $10800.00^{*}$ & 2250.00 & 175237.00 & 339.93 & -5.88 & -81.93 \\
\hline R206 & 100 & 10 & 174794.14 & 14150.00 & 187873.20 & $10800.00^{*}$ & 2250.00 & 175168.00 & 268.04 & -6.76 & -84.10 \\
\hline R207 & 100 & 10 & 174794.09 & 11950.00 & 185389.11 & $10800.00^{*}$ & 2250.00 & 175096.00 & 270.70 & -5.55 & -81.17 \\
\hline R208 & 100 & 10 & 174794.09 & 7500.00 & 180572.09 & $10800.00^{*}$ & 2250.00 & 175010.00 & 322.05 & -3.08 & -70.00 \\
\hline R209 & 100 & 10 & 174794.09 & 14700.00 & 188632.91 & $10800.00^{*}$ & 2250.00 & 175156.00 & 288.98 & -7.14 & -84.69 \\
\hline R210 & 100 & 10 & 174794.14 & 14850.00 & 188607.92 & $10800.00^{*}$ & 2250.00 & 175189.00 & 311.21 & -7.11 & -84.85 \\
\hline R211 & 100 & 10 & 174794.14 & 12200.00 & 185459.83 & $10800.00^{*}$ & 2250.00 & 175051.00 & 247.03 & -5.61 & -81.56 \\
\hline RC101 & 100 & 10 & 39180.69 & 3510.00 & 40800.29 & $10800.00^{*}$ & 3300.00 & 40111.60 & 323.52 & -1.69 & -5.98 \\
\hline RC102 & 100 & 10 & 39186.00 & 7530.00 & 44712.85 & $10800.00^{*}$ & 3330.00 & 39940.70 & 328.25 & -10.67 & -55.78 \\
\hline RC103 & 100 & 10 & 39199.13 & 7890.00 & 70169.17 & $10800.00^{*}$ & 3510.00 & 39828.90 & 316.82 & -43.24 & -55.51 \\
\hline RC104 & 100 & 10 & 39181.17 & 7530.00 & 67342.83 & $10800.00^{*}$ & 3420.00 & 39727.00 & 317.03 & -41.01 & -54.58 \\
\hline RC105 & 100 & 10 & 39203.16 & 4710.00 & 42222.87 & $10800.00^{*}$ & 3390.00 & 39990.10 & 281.31 & -5.29 & -28.03 \\
\hline RC106 & 100 & 10 & 39176.91 & & 42502.09 & $10800.00^{*}$ & 3420.00 & 39912.40 & 323.17 & -6.09 & -32.94 \\
\hline RC107 & 100 & 10 & 39176.91 & 6930.00 & 44066.07 & $10800.00^{*}$ & 3420.00 & 39827.50 & 275.89 & -9.62 & -50.65 \\
\hline RC108 & 100 & 10 & 39187.37 & 5790.00 & 42617.67 & $10800.00^{*}$ & 3450.00 & 39775.20 & 282.44 & -6.67 & -40.41 \\
\hline RC201 & 100 & 10 & 174428.16 & 4700.00 & 177634.48 & $10800.00^{*}$ & 3150.00 & 175461.00 & 282.26 & -1.22 & -32.98 \\
\hline RC202 & 100 & 10 & 174429.52 & 8500.00 & 181651.48 & $10800.00^{*}$ & 3100.00 & 175284.00 & 278.25 & -3.51 & -63.53 \\
\hline RC203 & 100 & 10 & 174428.48 & 11700.00 & 185169.73 & $10800.00^{*}$ & 3250.00 & 175271.00 & 313.55 & -5.35 & -72.22 \\
\hline RC204 & 100 & 10 & 174435.83 & 37550.00 & 873972.63 & $10800.00^{*}$ & 1350.00 & 173214.00 & 260.57 & -80.18 & -96.40 \\
\hline RC205 & 100 & 10 & 174432.47 & 9850.00 & 183169.93 & $10800.00^{*}$ & 3100.00 & 175409.00 & 317.51 & -4.24 & -68.53 \\
\hline RC206 & 100 & 10 & 174427.49 & 11600.00 & 184800.00 & $10800.00^{*}$ & 3150.00 & 175333.00 & 318.89 & -5.12 & -72.84 \\
\hline RC207 & 100 & 10 & 174426.83 & 6350.00 & 179681.12 & $10800.00^{*}$ & 3250.00 & 175280.00 & 300.42 & -2.45 & -48.82 \\
\hline RC208 & 100 & 10 & 174436.39 & 10300.00 & 183920.02 & $10800.00^{*}$ & 3350.00 & 175210.00 & 287.60 & -4.74 & -67.48 \\
\hline
\end{tabular}




\section{References}

Achuthan, N., Caccetta, L., Hill, S., 2003. An improved branch-and-cut algorithm for the capacitated vehicle routing problem. Transportation Science 37, 153-169.

Albareda-Sambola, M., Díaz, J. A., Fernández, E., 2005. A compact model and tight bounds for a combined location-routing problem. Computers \& Operations Research 32, 407-428.

Albareda-Sambola, M., 2015. Location-routing and location-arc routing problems. In Laporte, G., Nickel, S., Saldanha da Gama, F. (Eds.), Location Science. (pp. 399-418). Springer, Berlin-Heidelberg.

Ambrosino, D., Sciomachen, A., Scutellà, M. G., 2009. A heuristic based on multiexchange techniques for a regional fleet assignment location-routing problem, Computers \& Operations Research 36, 442-460.

Baldacci, R., Battarra, M., Vigo, D., 2008. Routing a heterogeneous fleet of vehicles. In Golden, B.L., Raghavan, S., Wasil, E.A. (Eds), The Vehicle Routing Problem: Latest Advances and New Challenges. (pp. 1-25). Springer, New York.

Baldacci, R., Mingozzi, A., 2009. A unified exact method for solving different classes of vehicle routing problems. Mathematical Programming 120, 347-380.

Balinski, M. L., 1965. Integer programming: Methods, uses, computation. Management Science 12, 253-313.

Barreto, S., 2004. Análise e modelização de problemas de localização-distribuição (Analysis and modelling of location-routing problems). Ph.D. thesis, University of Aveiro, Aveiro, Portugal.

Berger, R. T., Coullard, C. R., Daskin, M. S., 2007. Location-routing problems with distance constraints, Transportation Science 41, 29-43.

Bräysy, O., Dullaert, W., Hasle, G., Mester, D., Gendreau, M., 2008. An effective multirestart deterministic annealing metaheuristic for the fleet size and mix vehicle routing problem with time windows. Transportation Science 42, 371-386.

Bräysy, O., Porkka, P. P., Dullaert, W., Repoussis, P. P., Tarantilis, C. D., 2009. A well scalable metaheuristic for the fleet size and mix vehicle-routing problem with time windows. Expert System with Applications $36,8460-8475$.

Clarke, G., Wright, J. W., 1964. Scheduling of vehicles from a central depot to a number of delivery points. Operations Research 12, 568-581. 
Dantzig, G. B., Fulkerson, D. R., Johnson, S. M., 1954. Solution of a large-scale traveling salesman problem. Operations Research 2, 393-410.

Dell'Amico, M., Monaci, M., Pagani, C., Vigo, D., 2007. Heuristic approaches for the fleet size and mix vehicle routing problem with time windows. Transportation Science 41, 516-526.

Demir, E., Bektaş, T., Laporte, G., 2012. An adaptive large neighborhood search heuristic for the pollutionrouting problem. European Journal of Operational Research 223, 346-359.

Drexl, M., Schneider, M., 2015. A survey of variants and extensions of the location-routing problem. European Journal of Operational Research 241, 283-308.

Duhamel, C., Lacomme, P., Prins, C., Prodhon, C., 2010. A GRASPXELS approach for the capacitated location-routing problem. Computers \& Operations Research 37, 1912-1923.

Duhamel, C., Lacomme, P., Prodhon, C. 2011. Efficient frameworks for greedy split and new depth first search split procedures for routing problems. Computers \& Operations Research 38, 723-739.

Dullaert, W., Janssens, G. K., Sörensen, K., Vernimmen, B., 2002. New heuristics for the fleet size and mix vehicle routing problem with time windows. Journal of the Operational Research Society 53, 1232-1238.

Golden, B. L., Assad, A. A., Levy, L., Gheysens, F., 1984. The fleet size and mix vehicle routing problem. Computers \& Operations Research 11, 49-66.

Hemmelmayr, V. C., Cordeau, J.-F., Crainic, T. G., 2012. An adaptive large neighborhood search heuristic for two-echelon vehicle routing problems arising in city logistics. Computers \& Operations Research 39, 3215-3228.

Hoff, A., Andersson, H., Christiansen, M., Hasle, G., Løkketangen, A., 2010. Industrial aspects and literature survey: fleet composition and routing. Computers \& Operations Research 37, 2041-2061.

Irnich, S., Schneider, M., Vigo, D. 2014. Four variants of the vehicle routing problem, in P. Toth and D. Vigo, (Eds.), Vehicle Routing: Problems, Methods and Applications. (pp. 241-271). MOS-SIAM Series in Optimization, Philadelphia.

Karaoglan I., Altiparmak F., Kara I., Dengiz B., 2011. A branch and cut algorithm for the location-routing problem with simultaneous pickup and delivery. European Journal of Operational Research 211, 318-332.

Karaoglan I., Altiparmak F., Kara I., Dengiz B., 2012. The location-routing problem with simultaneous pickup and delivery: Formulations and a heuristic approach. Omega 40, 465-477. 
Koç, Ç., Bektaş, T., Jabali, O., Laporte, G., 2014. The fleet size and mix pollution-routing problem. Transportation Research Part B: Methodological 70, 239-254.

Koç, Ç., Bektaş, T., Jabali, O., Laporte, G., 2015. A hybrid evolutionary algorithm for heterogeneous fleet vehicle routing problems. Computers \& Operations Research 64, 11-27.

Labbé, M., Rodríguez-Martin, I., Salazar-González, J. J., 2004. A branch-and-cut algorithm for the plantcycle location problem. Journal of the Operational Research Society 55, 513-520.

Laporte, G., 1988. Location-routing problems. In: Golden, B.L., Assad, A.A. (Eds.), Vehicle Routing: Methods and Studies. North-Holland, Amsterdam, pp. 163-198.

Liu, F. H., S. Y. Shen. 1999. The fleet size and mix vehicle routing problem with time windows. Journal of the Operational Research Society 50, 721-732.

Min, H., Jayaraman, V., Srivastava, R., 1998. Combined location-routing problems: A synthesis and future research directions. European Journal of Operational Research 108, 1-15.

Nagata, Y., Bräysy, O., Dullaert, W., 2010. A penalty-based edge assembly memetic algorithm for the vehicle routing problem with time windows. Computers \& Operations Research 37, 724-737.

Nagy, G., Salhi, S. 2007. Location-routing: Issues, models and methods. European Journal of Operational Research 177, 649-672.

Prins, C., Prodhon, C., Wolfler Calvo, R., 2006a. Solving the capacitated locationrouting problem by a GRASP complemented by a learning process and a path relinking. 4OR: A Quarterly Journal of Operations Research 4, 221-238.

Prins, C., Prodhon, C., Wolfler Calvo, R., 2006b. A memetic algorithm with population management (MAj,PM) for the capacitated location-routing problem. In J. Gottlieb G. R. Raidl (Eds.), Evolutionary computation in combinatorial optimization. Lecture notes in computer science, Vol. 3906, pp. 183-194. Berlin Heidelberg: Springer.

Prodhon, C. 2006. Le problème de localisation-routage (The location-routing problem). Ph.D. thesis, Troyes University of Technology, Troyes, France.

Prodhon, C. Prins, C. 2014. A survey of recent research on location-routing problems. European Journal of Operational Research 238, 1-17. 
Repoussis, P. P., Tarantilis, C. D., 2010. Solving the fleet size and mix vehicle routing problem with time windows via adaptive memory programming. Transportation Research Part C: Emerging Technologies $18,695-712$.

Ropke, S., Pisinger, D., 2006a. An adaptive large neighborhood search heuristic for the pickup and delivery problem with time windows. Transportation Science 40, 455-472.

Ropke, S., Pisinger, D., 2006b. A unified heuristic for a large class of vehicle routing problems with backhauls. European Journal of Operational Research 171,750-775.

Solomon, M. M. 1987. Algorithms for the vehicle routing and scheduling problems with time window constraints. Operations Research 35, 254-265.

Taillard, É.D., 1999. A heuristic column generation method for the heterogeneous fleet vehicle routing problem. RAIRO (Recherche Opérationnelle / Operations Research) 33, 1-14.

Vidal, T., Crainic, T.G., Gendreau, M., Prins, C., 2014. A unified solution framework for multi-attribute vehicle routing problems. European Journal of Operational Research 234, 658-673.

Von Boventer, E. 1961. The relationship between transportation costs and location rent in transportation problems. Journal of Regional Science 3, 27-40.

Wu, T.H., Low, C., Bai J. W., 2002. Heuristic solutions to multi-depot location-routing problems. Computers \& Operations Research 29, 1393-415. 